\title{
Thermobarometry of mantle-derived garnets and pyroxenes of Kola region (NW Russia): lithosphere composition, thermal regime and diamond prospectivity
}

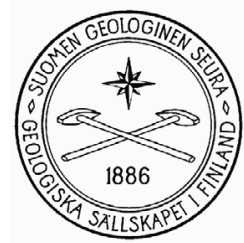

\author{
Dmitry R. Zozulya ${ }^{1)}$, Hugh O’Brien ${ }^{2)^{*}}$, Petri Peltonen ${ }^{3)}$, and Marja \\ LEHTONEN $^{2)}$ \\ ${ }^{1)}$ Geological Institute, Kola Science Centre, 14 Fersman St., 184209 Apatity, Russia \\ ${ }^{2)}$ Geological Survey of Finland, P.O. Box 96, FI-02151, Espoo, Finland \\ 3) Northland Exploration Finland Oy, Ahventie 4, FI-02170, Espoo, Finland
}

\begin{abstract}
More than 700 pyrope, chrome diopside and chromite grains recovered from Quaternary sediments of the Kola craton, and from the Ermakovsky kimberlite in the Terskii Coast field were analyzed in order to determine their P-T parameters. Ni-thermometry on pyropes from SE Kola gives a range of temperatures between $650-1250{ }^{\circ} \mathrm{C}$, corresponding to a sampling interval of c. $75-190 \mathrm{~km}$. Pyrope compositions imply a stratified structure to the SE Kola lithospheric mantle, with GI0-pyropes absent in the shallow mantle (75-110 km) where the bulk of the G9-pyropes are sourced, while a deeper mantle horizon, between I 10 and $190 \mathrm{~km}$, has contributed abundant GI0-pyropes. Approximately $16 \%$ of all these pyropes are derived from the diamond stability field. The Ni-temperatures of garnets from the Ermakovsky kimberlite reveal a relatively shallow sampling interval of the mantle (c. 75-140 km), dominantly within the graphite-stability field. P-T values for peridotitic chrome diopsides imply that most grains from SE Kola were derived from the graphite stability field, apparently originating from non-diamondiferous alkaline-ultramafic dykes. Nevertheless, c. $15 \%$ of SE Kola diopsides have been derived from the stability field of diamond. Diopsides from SW Kola mostly plot in the diamond stability field. 20 \% of diopsides from central Kola are derived from the diamond stability field, whereas diopsides from northern Kola region all fall within the stability field of graphite. The maximum depth of diopside xenocryst sampling varies from up to $200 \mathrm{~km}$ in SE and SW Kola, to $170 \mathrm{~km}$ in central Kola, and only to $140 \mathrm{~km}$ in the northern Kola region. The P-T values for chrome diopsides imply significant regional differences in heat flow: $38-44 \mathrm{~mW} / \mathrm{m}^{2}$ within the southern part of Kola adjacent to the Kandalaksha graben; $35-38 \mathrm{~mW} / \mathrm{m}^{2}$ towards the SE and $\mathrm{SW}$ away from the graben; 38-44 mW/m² in central Kola; and up to $50 \mathrm{~mW} / \mathrm{m}^{2}$ in northernmost Kola. These data indicate that the more "cratonic" SE, SW and likely central parts of the Kola region have higher potential for diamondiferous kimberlitic magmatism.
\end{abstract}

Key words: Kola Peninsula, lithosphere, mantle, pyrope, diopside, kimberlite

*Corresponding author email: hugh.obrien@gtk.fı 


\section{Introduction}

The Kola region of the eastern Fennoscandian shield is prospective for diamondiferous magmatism based on structural-tectonic conditions that include: confinement to a platform with a Precambrian basement, thick lithosphere (170-240 km), and low heat flow $\left(<40 \mathrm{~mW} / \mathrm{m}^{2}\right)$. It is located within the Kola and Karelian cratons, which were amalgamated during the Late Archean. Kimberlitic magmatism, another factor critical for diamond prospectivity, has been recognized in the Kola region, occurring as abundant dykes and explosive pipes of alkaline and alkaline-ultrabasic composition (Fig. 1). Numerous kimberlit- ic indicator minerals and diamonds have been recovered from Quaternary sediments of the region. Based on these discoveries, kimberlitic fields are predicted in the Makeevka, Pyalitsa, Pulonga and Snezhnitsa fields in south-east Kola and the Zarechensk field in south-west Kola (Gavilenko et al., 2000; Zozulya et al., 2008). Also prospective is the southern part of region where the two Ermakovsky low-grade kimberlitic pipes are located (Kalinkin et al., 1993; Beard et al., 1998).

The main purpose of this study is to elucidate the composition and thermal properties of the lithospheric mantle beneath the Kola region and estimate its diamond prospectivity. For this, the chemical com-

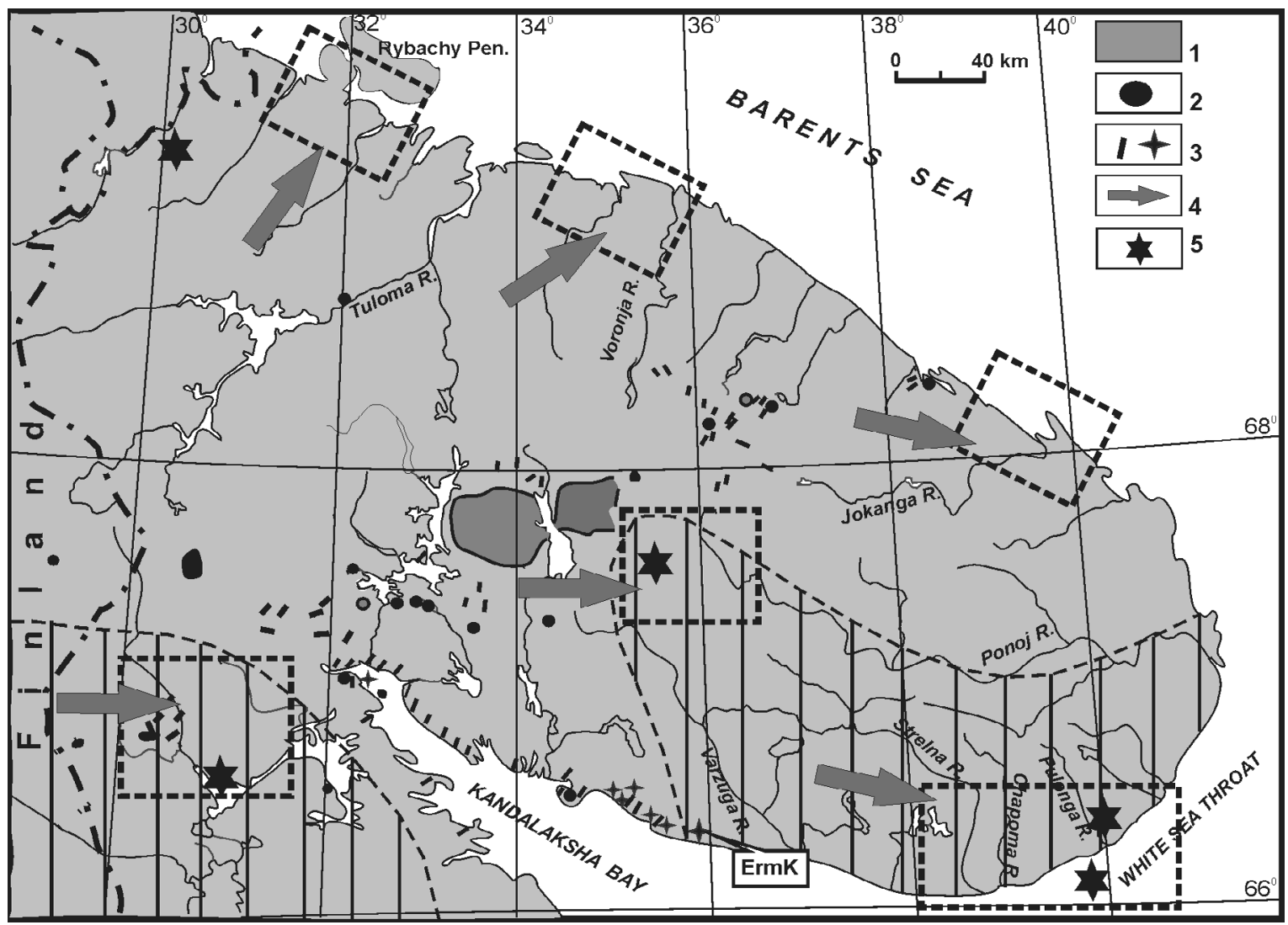

Fig. I. Location of mantle-derived magmatic rocks in the Kola craton and the areas sampled (boxes) from the south-eastern, south-western, central and northern parts of the region. $I=$ nepheline syenites, $2=$ alkaline-ultrabasic rocks and carbonatites, $3=$ alkaline-ultrabasic dykes and pipes, $4=$ main transportation direction of Quaternary material by the Scandinavian ice-sheet, 5 = diamond discoveries in Quaternary sediments. ErmK = Ermakovsky kimberlitic pipe in the Terskii Coast field. Striped areas on map are prospective for diamondiferous kimberlites based on this study. 
positions of mantle-derived garnets and pyroxenes were determined and P-T parameters were calculated allowing discrimination of mantle-derived minerals. Previously obtained P-T values for mantle xenoliths (eclogitic and peridotitic) from alkaline dykes and pipes of the region vary between $10-15 \mathrm{kbar}$ and 775-1050 ㅇ (Arzamastsev et al., 1988; Vetrin \& Kalinkin, 1992; Beard et al., 2007).

\section{Sampling}

More than 600 pyrope and chrome diopside grains recovered from Quaternary sediments (till, alluvium and coastal sediments) of the southern, central and northern parts of the Kola Craton (Fig. 1) were analyzed. Representing either mantle xenocrysts or constituents of mantle xenoliths, the pyropes and chrome diopsides contain valuable information on the composition of the lithospheric mantle and its thermal properties. It has been established (Hirvas, 1977) that in glaciated areas, the main proportion $(75-90 \%)$ of transported detrital material has local $(0-5 \mathrm{~km})$ rather than distal sources. This implies that the samples in this study mostly represent locally derived materials. These sediments also contain pyropes and diopsides from relatively shallow crustal eclogites and eclogitic xenoliths and xenocrysts from non-kimberlitic dykes and pipes. Consequently a thorough discrimination must be made to exclude any grains not originally derived from deep lithospheric peridotites.

The glaciated south-western, south-eastern and central sampling areas were affected by the Scandinavian ice-sheet that transported detrital material from west to east (Fig. 1). In the northern sampling areas lobes of the same ice-sheet transported material to the north and north-east (Hattestrand et al., 2007, and references therein). The south-western and southeastern areas are far from the Kandalaksha graben, which represents a Belomorian rift system with abundant Devonian alkaline-mafic dyke and pipe magmatism on its flanks (Arzamastsev \& Dalgren, 1993; Beard et al., 1996; Beard et al., 2007; Downes et al., 2005; Vetrin \& Kalinkin, 1992). The bedrock in these areas is dominantly Late Archean with minor Pale- proterozoic units of the Karelian and Kola cratons, respectively (Mitrofanov, 1996). The central sampling area is located south-east of the Lovozero nepheline syenite massif where the bedrock is represented mostly by Late Archean TTG-basement. The northern sampling areas are located in the vicinity of the Barents Sea, on- and offshore of Kola craton and confined spatially to the flanks of a graben of the Barents rift system (Baluev, 2006). Here the bedrock is represented mostly by Late Archean and less abundant Late Proterozoic rocks. Scarse alkaline-ultrabasic dykes and pipes have been found in some of the northern areas (Arzamastsev \& Dalgren, 1993).

Pyrope and chrome diopside were recovered from sediments while sluicing for diamonds and kimberlitic indicator minerals in Quaternary sediments of the Kola region. In total, more than 500 samples of an initial volume of $0.02 \mathrm{~m}^{3}$ were processed in the field by sieving to $<4 \mathrm{~mm}$ grain size and sluicing in a pan to produce heavy mineral concentrates (gray sand). The heavy concentrates mostly consisted of garnet, pyroxene, amphibole, epidote (total 80-90 vol.\%); magnetite (1-5\%); kyanite, sillimanite, staurolite, biotite, apatite (each mineral up to $1 \%$ ); rutile, perovskite, zircon, hematite and loparite (dozens of grains).

The heavy mineral concentrates were processed using an electromagnetic separator, and KIM's were handpicked from the subsequent fractions, as follows: (a) a sample was first separated using a low electromagnetic current (Cr-spinels were recovered from the magnetic fraction and pyropes from the non-magnetic fraction), (b) the non-magnetic fraction from the previous run was treated again using a high electromagnetic current to produce a magnetic fraction from which pyropes and part of the $\mathrm{Cr}$ diopsides were recovered, and a non magnetic fraction containing diamonds and the remaining $\mathrm{Cr}$ diopsides.

The $>80$ pyrope grains reported here are nearly completely confined to the south-eastern sampling area, being very scarse in samples from other areas. Chrome-diopsides are most abundant and widespread ( -350 grains from the south-eastern area, -100 grains from the central area, -100 grains from the northern areas combined, and -20 grains from 
the south-western area). Diamonds are found as single grains in south-eastern, south-western and central areas (Fig. 1). The location of the sampled areas and the main direction of detrital material transportation make it impossible that any of the indicator minerals were derived from the Arkhangelsk kimberlitic province and the Kaavi-Kuopio kimberlites (however the Kuusamo field in Northern Finland could be regarded as a possible source for the south-western sampling area).

The pyropes from Ermakovsky kimberlite, Terskii Coast field in southern Kola region, were recovered from a $\sim 20 \mathrm{~kg}$ representative sample of phlogopite-olivine diatreme-facies rock. The Ermakovsky low grade pipes are comparatively small $(30 \times 40 \mathrm{~m}$ and 130 $\mathrm{x} 130 \mathrm{~m}$ ) and are mostly diatreme facies, with some crater facies preserved. Age estimates for the Terskii kimberlites range from 360 to $380 \mathrm{Ma}$ (Kalinkin et al., 1993) to c. $460 \mathrm{Ma}$ (Delenitzin et al., 2001.). By chemical and mineralogical composition these kimberlites are most similar to Group I kimberlites of the Koidu field in West Africa and the Kaavi-Kuopio field in Finland (Beard et al., 1998). Mantle xenoliths are not found in Ermakovsky kimberlite and pyropes apparently occur as xenocrysts. A total of 39 pyrope grains were recovered and analyzed.

\section{Analytical methods}

Garnet major element compositions were determined by a Cameca Camebax SX100 electron microprobe at the GTK E-beam Laboratory, applying an acceleration voltage of $25 \mathrm{kV}$, probe current of $48 \mathrm{nA}$ and beam diameter of $1 \mu \mathrm{m}$. The parameters for clinopyroxene analyses were $15 \mathrm{kV}, 30 \mathrm{nA}$ and $5 \mu \mathrm{m}$, respectively. Trace Ni data were obtained on pyrope grains by a Cameca Camebax SX50 electron microprobe at the GTK E-beam Laboratory employing 500 nA probe current, 600s counting times on peak plus background positions with data reduction by the CSIRO TRACE program (Robinson \& Graham, 1992).

A small proportion of pyroxene (c. $10 \%$ ) analyses were made by a Cameca MS-46 electron microprobe (EMP) at the Geological Institute of the Kola
Science Centre, applying an acceleration voltage of $22 \mathrm{kV}$, probe current 20 to $40 \mathrm{nA}$ and beam diameter of 2 to $5 \mu \mathrm{m}$.

Representative chemical analyses of garnets and pyroxenes from the Kola region are listed in Tables 1,2 and 3 .

\section{Results}

\section{I Mineral chemistry and mantle source rocks of the garnets}

Garnet chemical and genetic classification was performed mainly using the distribution of $\mathrm{Cr}_{2} \mathrm{O}_{3}, \mathrm{CaO}$, $\mathrm{MgO}$ and $\mathrm{TiO}_{2}$. Pyropes from the south-eastern part of the Kola region show the following variations: $\mathrm{Cr}_{2} \mathrm{O}_{3}(0.1-11$ wt.\%), $\mathrm{CaO}$ (1.2-9.0 wt.\%), $\mathrm{MgO}$ (10-22 wt.\%) and $\mathrm{TiO}_{2}(0-0.4$ wt.\%). Based on commonly used classification systems (Sobolev et al., 1973; Sobolev, 1974; Gurney, 1984) it is established that $45 \%$ of the total quantity of pyropes belong to the lherzolite association (G9, Fig. 2a). Importantly, the abundance of pyropes of the potentially diamondiferous low-Ca harzburgite association (G10) is high, c. $30 \%$. For most kimberlitic occurrences G10 pyropes rarely exceeds $10 \%$, or at a maximum $20 \%$. Apparently this distribution of G10-G9 pyropes is related to the specific composition of the Kola lithospheric mantle. Moreover, no wehrlitic pyropes were found in this collection, as is also true for the kimberlites of the Arkhangelsk province (Bogatikov, 2000). The diamond-associated pyropes (inclusions in diamonds) are dominantly G10s with $\mathrm{Cr}_{2} \mathrm{O}_{3}>5 \%$, $\mathrm{CaO}=0-7.4 \%$ (Sobolev, 1974). There are about $9 \%$ of such pyropes in the south-eastern Kola collection. One of the mineralogical criteria for a kimberlite to be economically important is that the number of diamond-associated pyropes should be at least $5 \%$ of the total amount (Sobolev, 1974). Coupled with the fact that G9 and G10 pyropes are mainly of kimberlitic origin, the presence of a large number of diamond-associated pyropes in south-eastern Kola collection suggests its high diamond prospectivity.

Approximately $25 \%$ of pyropes from the collection belong to the G3 group (pyrope-almandine) 
Table I. Chemical composition of pyropes from the Quaternary sediments of the SE Kola region (wt.\%)

\begin{tabular}{|c|c|c|c|c|c|c|c|c|c|c|c|c|}
\hline Sample & B-12-04 & $\begin{array}{c}\text { MX- } \\
19.02 \mathrm{~A}\end{array}$ & $\begin{array}{c}\text { MX- } \\
19.02 \mathrm{C}\end{array}$ & $\begin{array}{c}\text { PL- } \\
22.02\end{array}$ & $\begin{array}{c}\text { PL- } \\
22.02 B\end{array}$ & $\begin{array}{c}\text { PL- } \\
24.02\end{array}$ & PL27/2 & PL29/1 & PL4.02a & PL4.02c & PR1 & PR4 \\
\hline $\mathrm{SiO}_{2}$ & 42.10 & 40.52 & 41.58 & 40.98 & 40.74 & 40.37 & 40.78 & 40.74 & 41.43 & 41.33 & 41.03 & 40.91 \\
\hline $\mathrm{TiO}_{2}$ & 0.41 & 0.16 & 0.00 & 0.09 & 0.33 & 0.01 & 0.07 & 0.19 & 0.11 & 0.03 & 0.02 & 0.23 \\
\hline $\mathrm{Al}_{2} \mathrm{O}_{3}$ & 18.60 & 17.47 & 22.72 & 17.60 & 19.05 & 17.87 & 16.77 & 19.80 & 20.10 & 19.89 & 16.93 & 19.58 \\
\hline $\mathrm{Cr}_{2} \mathrm{O}_{3}$ & 7.57 & 8.63 & 2.13 & 8.58 & 6.23 & 8.60 & 9.49 & 5.52 & 5.76 & 6.18 & 9.83 & 5.60 \\
\hline $\mathrm{V}_{2} \mathrm{O}_{3}$ & 0.02 & 0.04 & 0.01 & 0.04 & 0.07 & 0.02 & 0.07 & 0.04 & 0.03 & 0.04 & 0.06 & 0.03 \\
\hline $\mathrm{FeO}$ & 8.07 & 6.88 & 7.18 & 5.75 & 6.26 & 7.24 & 5.83 & 7.06 & 5.90 & 6.82 & 6.50 & 6.76 \\
\hline $\mathrm{MnO}$ & 0.41 & 0.38 & 0.40 & 0.27 & 0.30 & 0.47 & 0.27 & 0.36 & 0.36 & 0.38 & 0.37 & 0.37 \\
\hline $\mathrm{MgO}$ & 20.70 & 20.27 & 22.03 & 22.45 & 21.13 & 20.45 & 21.17 & 20.72 & 22.04 & 22.09 & 23.78 & 20.83 \\
\hline $\mathrm{CaO}$ & 4.96 & 5.29 & 3.52 & 3.57 & 5.09 & 4.40 & 5.54 & 4.82 & 4.57 & 3.75 & 1.24 & 4.81 \\
\hline $\mathrm{Na}_{2} \mathrm{O}$ & 0.08 & 0.04 & 0.02 & 0.02 & 0.07 & 0.01 & 0.00 & 0.05 & 0.03 & 0.01 & 0.01 & 0.05 \\
\hline Total & 102.92 & 99.68 & 99.59 & 99.35 & 99.27 & 99.44 & 99.99 & 99.30 & 100.33 & 100.52 & 99.77 & 99.17 \\
\hline $\begin{array}{l}\text { Garnet } \\
\text { group }\end{array}$ & G-10 & G-10 & G-10 & G-10 & G-10 & G-10 & G-10 & G-10 & G-10 & G-10 & G-10 & G-10 \\
\hline $\mathrm{Mg} \#$ & 0.82 & 0.84 & 0.85 & 0.87 & 0.86 & 0.83 & 0.87 & 0.84 & 0.87 & 0.85 & 0.87 & 0.85 \\
\hline $\mathrm{Ni}, \mathrm{ppm}$ & 35 & 40 & 16 & 77 & 50 & 30 & 81 & 41 & 29 & 27 & 42 & 29 \\
\hline $\mathrm{Mn}, \mathrm{ppm}$ & 2953 & 2862 & 2825 & 1999 & 2314 & 3536 & 1980 & 2813 & 2554 & 2863 & 2758 & 2776 \\
\hline $\mathrm{Ti}, \mathrm{ppm}$ & 1912 & 849 & 15 & 456 & 1847 & 63 & 318 & 946 & 500 & 151 & 172 & 1146 \\
\hline $\mathrm{T}(\mathrm{Ni}),{ }^{\circ} \mathrm{C}$ & 925 & 959 & 738 & 1184 & 1031 & 879 & 1207 & 966 & 876 & 859 & 977 & 876 \\
\hline Depth, km & 125 & 132 & 90 & 182 & 147 & 116 & 187 & 134 & 116 & 112 & 136 & 116 \\
\hline
\end{tabular}

\begin{tabular}{lrrrrrrrrrrrrr}
\hline Sample & PR67 & Sk-5b & B-01-04 & BL-23/1 & BL-3 & $\begin{array}{r}\text { MT- } \\
3-3\end{array}$ & $\begin{array}{c}\text { MX- } \\
19.02 B\end{array}$ & $\begin{array}{c}\text { MX- } \\
19.02 \mathrm{D}\end{array}$ & $\begin{array}{c}\text { MX- } \\
19.02 \mathrm{E}\end{array}$ & $\begin{array}{c}\text { MX- } \\
19.02 \mathrm{~F}\end{array}$ & $\begin{array}{c}\text { PL- } \\
15.02\end{array}$ & PL16.02 & PL- \\
\hline $\mathrm{SiO}_{2}$ & 40.86 & 40.20 & 40.83 & 41.52 & 40.96 & 39.93 & 41.49 & 41.24 & 41.10 & 41.63 & 41.33 & 41.94 & 41.31 \\
$\mathrm{TiO}_{2}$ & 0.00 & 0.02 & 0.02 & 0.04 & 0.12 & 0.16 & 0.36 & 0.03 & 0.16 & 0.01 & 0.37 & 0.06 & 0.34 \\
$\mathrm{Al}_{2} \mathrm{O}_{3}$ & 17.88 & 16.89 & 21.20 & 20.21 & 18.50 & 15.86 & 19.93 & 19.65 & 21.24 & 21.75 & 19.91 & 21.55 & 19.30 \\
$\mathrm{Cr}_{2} \mathrm{O}_{3}$ & 8.72 & 9.95 & 3.70 & 5.53 & 7.64 & 10.74 & 5.33 & 5.82 & 3.87 & 3.58 & 5.33 & 4.16 & 6.25 \\
$\mathrm{~V}_{2} \mathrm{O}_{3}$ & 0.05 & 0.05 & 0.02 & 0.02 & 0.05 & 0.03 & 0.04 & 0.04 & 0.02 & 0.02 & 0.07 & 0.02 & 0.06 \\
$\mathrm{FeO}$ & 7.21 & 6.46 & 7.16 & 7.48 & 7.23 & 7.15 & 6.31 & 8.68 & 7.94 & 7.00 & 6.32 & 6.87 & 6.34 \\
$\mathrm{MnO}$ & 0.45 & 0.36 & 0.38 & 0.45 & 0.41 & 0.46 & 0.27 & 0.52 & 0.47 & 0.39 & 0.28 & 0.36 & 0.32 \\
$\mathrm{MgO}$ & 22.05 & 23.58 & 19.54 & 20.31 & 19.71 & 18.20 & 21.97 & 17.88 & 20.41 & 20.26 & 21.69 & 20.51 & 21.04 \\
$\mathrm{CaO}$ & 2.66 & 1.21 & 5.84 & 5.33 & 5.97 & 6.95 & 5.00 & 7.13 & 4.80 & 5.96 & 5.01 & 5.77 & 5.15 \\
$\mathrm{Na}{ }_{2} \mathrm{O}$ & 0.02 & 0.02 & 0.00 & 0.01 & 0.02 & 0.04 & 0.03 & 0.01 & 0.04 & 0.00 & 0.05 & 0.02 & 0.04 \\
\hline $\mathrm{Total}$ & 99.90 & 98.74 & 98.69 & 100.90 & 100.61 & 99.52 & 100.73 & 101.00 & 100.05 & 100.60 & 100.36 & 101.26 & 100.15 \\
\hline $\mathrm{Garnet}$ & & & & & & & & & & & & & \\
group & $\mathrm{G}-10$ & $\mathrm{G}-10$ & $\mathrm{G}-9$ & $\mathrm{G}-9$ & $\mathrm{G}-9$ & $\mathrm{G}-9$ & $\mathrm{G}-9$ & $\mathrm{G}-9$ & $\mathrm{G}-9$ & $\mathrm{G}-9$ & $\mathrm{G}-9$ & $\mathrm{G}-9$ & $\mathrm{G}-9$ \\
$\mathrm{Mg} \#$ & 0.84 & 0.87 & 0.83 & 0.83 & 0.83 & 0.82 & 0.86 & 0.79 & 0.82 & 0.84 & 0.86 & 0.84 & 0.86 \\
$\mathrm{Ni}, \mathrm{pmm}$ & 28 & 43 & 16 & 28 & 31 & 30 & 66 & 20 & 13 & 14 & 20 & 12 & 30 \\
$\mathrm{Mn}, \mathrm{ppm}$ & 3217 & 2807 & 2683 & 3295 & 3097 & 3390 & 2168 & 3940 & 3456 & 3686 & 3051 & 2795 & 3466 \\
$\mathrm{Ti}, \mathrm{ppm}$ & 64 & 175 & 98 & 171 & 665 & 964 & 1894 & 184 & 913 & 396 & 5 & 329 & 63 \\
$\mathrm{~T}(\mathrm{Ni}),{ }^{\circ} \mathrm{C}$ & 863 & 984 & 745 & 863 & 893 & 885 & 1127 & 785 & 704 & 719 & 785 & 684 & 885 \\
$\mathrm{Depth}, \mathrm{km}$ & 113 & 138 & 91 & 113 & 119 & 117 & 169 & 98 & 83 & 86 & 98 & 80 & 117 \\
\hline
\end{tabular}


Table I. cont. Chemical composition of pyropes from the Quaternary sediments of the SE Kola region (wt.\%)

\begin{tabular}{lrrrrrrrrrrrrrr}
\hline Sample & PL27/1 & PL27/1a & PL29/2 & PL4.02b & PP-2.02 & PP-5.02 & PP-8.02 & PR10 & PR106 & PR109 & PR3 & R-1.1.02 & Sk-9b \\
\hline $\mathrm{SiO}_{2}$ & 40.75 & 40.75 & 41.19 & 41.26 & 41.17 & 40.83 & 41.63 & 40.97 & 41.41 & 41.21 & 40.86 & 41.09 & 40.62 \\
$\mathrm{TiO}_{2}$ & 0.00 & 0.00 & 0.00 & 0.06 & 0.05 & 0.07 & 0.25 & 0.00 & 0.23 & 0.04 & 0.10 & 0.08 & 0.01 \\
$\mathrm{Al}_{2} \mathrm{O}_{3}$ & 21.64 & 21.52 & 22.05 & 21.39 & 21.26 & 21.14 & 20.77 & 21.37 & 19.52 & 22.84 & 21.37 & 20.72 & 20.22 \\
$\mathrm{Cr}_{2} \mathrm{O}_{3}$ & 3.35 & 3.44 & 2.89 & 3.45 & 3.89 & 4.17 & 4.43 & 3.47 & 5.46 & 1.38 & 3.33 & 4.55 & 5.09 \\
$\mathrm{~V}_{2} \mathrm{O}_{3}$ & 0.02 & 0.01 & 0.01 & 0.02 & 0.03 & 0.02 & 0.04 & 0.01 & 0.04 & 0.01 & 0.01 & 0.04 & 0.06 \\
$\mathrm{FeO}$ & 7.07 & 7.11 & 7.31 & 8.60 & 8.60 & 8.02 & 7.57 & 6.74 & 6.16 & 9.03 & 8.66 & 7.95 & 7.71 \\
$\mathrm{MnO}$ & 0.40 & 0.37 & 0.44 & 0.55 & 0.52 & 0.52 & 0.43 & 0.35 & 0.27 & 0.41 & 0.48 & 0.46 & 0.40 \\
$\mathrm{MgO}$ & 20.29 & 20.43 & 20.27 & 18.71 & 19.46 & 19.35 & 20.87 & 20.38 & 21.65 & 19.70 & 19.03 & 19.98 & 19.74 \\
$\mathrm{CaO}$ & 5.37 & 5.38 & 5.45 & 6.13 & 5.45 & 5.70 & 4.66 & 5.33 & 4.97 & 4.77 & 5.54 & 5.45 & 5.45 \\
$\mathrm{Na}_{2} \mathrm{O}$ & 0.02 & 0.03 & 0.02 & 0.00 & 0.00 & 0.02 & 0.06 & 0.02 & 0.04 & 0.01 & 0.02 & 0.01 & 0.02 \\
\hline $\mathrm{Total}$ & 98.91 & 99.04 & 99.63 & 100.17 & 100.43 & 99.84 & 100.71 & 98.64 & 99.75 & 99.40 & 99.40 & 100.33 & 99.32 \\
\hline $\mathrm{Garnet}$ & & & & & & & & & & & & & \\
group & $\mathrm{G}-9$ & $\mathrm{G}-9$ & $\mathrm{G}-9$ & $\mathrm{G}-9$ & $\mathrm{G}-9$ & $\mathrm{G}-9$ & $\mathrm{G}-9$ & $\mathrm{G}-9$ & $\mathrm{G}-9$ & $\mathrm{G}-9$ & $\mathrm{G}-9$ & $\mathrm{G}-9$ & $\mathrm{G}-9$ \\
$\mathrm{Mg} \#$ & 0.84 & 0.84 & 0.83 & 0.79 & 0.80 & 0.81 & 0.83 & 0.84 & 0.86 & 0.80 & 0.80 & 0.82 & 0.82 \\
$\mathrm{Ni}$, ppm & 20 & 84 & 21 & 15 & 17 & 16 & 26 & 25 & 82 & 18 & 18 & 27 & 17 \\
$\mathrm{Mn}, \mathrm{ppm}$ & 2788 & 1982 & 3276 & 4075 & 3743 & 3649 & 3134 & 2524 & 1960 & 3115 & 3440 & 3307 & 3079 \\
$\mathrm{Ti}$, ppm & 10 & 322 & 25 & 339 & 298 & 400 & 1323 & 9 & 1301 & 272 & 549 & 417 & 52 \\
$\mathrm{~T}(\mathrm{Ni}),{ }^{\circ} \mathrm{C}$ & 785 & 1222 & 795 & 732 & 750 & 745 & 845 & 836 & 1212 & 768 & 762 & 859 & 757 \\
Depth, km & 98 & 191 & 100 & 88 & 92 & 91 & 110 & 108 & 189 & 95 & 94 & 112 & 93 \\
\hline
\end{tabular}

\begin{tabular}{|c|c|c|c|c|c|c|c|c|c|c|c|c|c|}
\hline Sample & 12 & 62 & 34 & 38 & 33 & 14 & 16 & 19 & 22 & $4-2$ & PL4/1 & PL4/6 & PL4/2 \\
\hline $\mathrm{SiO}_{2}$ & 39.95 & 40.38 & 41.42 & 41.34 & 39.36 & 40.46 & 40.34 & 40.25 & 40.31 & 40.30 & 39.22 & 39.56 & 39.51 \\
\hline $\mathrm{TiO}_{2}$ & 0.00 & 0.00 & 0.07 & 0.13 & 0.20 & 0.06 & 0.13 & 0.06 & 0.00 & 0.14 & 0.13 & 0.08 & 0.16 \\
\hline $\mathrm{Al}_{2} \mathrm{O}_{3}^{2}$ & 20.70 & 21.03 & 21.57 & 21.31 & 21.35 & 21.96 & 21.72 & 21.87 & 21.83 & 22.15 & 22.45 & 22.72 & 22.38 \\
\hline $\mathrm{Cr}_{2} \mathrm{O}_{3}$ & 0.08 & 0.10 & 0.04 & 0.07 & 0.11 & 0.09 & 0.04 & 0.03 & 0.07 & 0.00 & 0.15 & 0.00 & 0.13 \\
\hline $\mathrm{V}_{2} \mathrm{O}_{3}$ & & & & & & & & & & & 0.01 & 0.01 & 0.00 \\
\hline $\mathrm{FeO}$ & 16.59 & 15.65 & 12.62 & 14.38 & 17.46 & 14.28 & 14.69 & 13.20 & 18.48 & 17.07 & 17.15 & 14.29 & 16.34 \\
\hline $\mathrm{MnO}$ & 0.14 & 0.17 & 0.35 & 0.31 & 0.42 & 0.38 & 0.19 & 0.10 & 0.99 & 0.41 & 0.50 & 0.17 & 0.40 \\
\hline $\mathrm{MgO}$ & 14.84 & 15.39 & 18.61 & 16.28 & 14.60 & 15.25 & 13.23 & 16.36 & 13.60 & 12.90 & 13.61 & 13.49 & 14.07 \\
\hline $\mathrm{CaO}$ & 6.01 & 6.38 & 5.05 & 5.70 & 6.01 & 7.56 & 9.01 & 7.82 & 5.18 & 6.15 & 5.19 & 8.23 & 5.45 \\
\hline $\mathrm{Na}_{2} \mathrm{O}$ & & & & & & & & & & & 0.05 & 0.01 & 0.01 \\
\hline Total & 98.31 & 99.10 & 99.73 & 99.52 & 99.51 & 100.04 & 99.34 & 99.69 & 100.46 & 99.12 & 98.46 & 98.56 & 98.45 \\
\hline $\begin{array}{l}\text { Garnet } \\
\text { group }\end{array}$ & G-3 & G-3 & G-3 & G-3 & G-3 & G-3 & G-3 & G-3 & G-3 & G-3 & G-3 & G-3 & G-3 \\
\hline $\mathrm{Mg} \#$ & 0.61 & 0.64 & 0.72 & 0.67 & 0.60 & 0.66 & 0.62 & 0.69 & 0.57 & 0.57 & 0.59 & 0.63 & 0.61 \\
\hline $\mathrm{Ni}, \mathrm{ppm}$ & & & & & & & & & & & 17 & 75 & 32 \\
\hline $\mathrm{Mn}, \mathrm{ppm}$ & & & & & & & & & & & 3552 & 1340 & 2951 \\
\hline $\mathrm{Ti}, \mathrm{ppm}$ & & & & & & & & & & & 677 & 400 & 906 \\
\hline
\end{tabular}


Table 2. Chemical composition of pyropes from Ermakovsky kimberlite, Terskii Coast field of the southern Kola region (wt.\%)

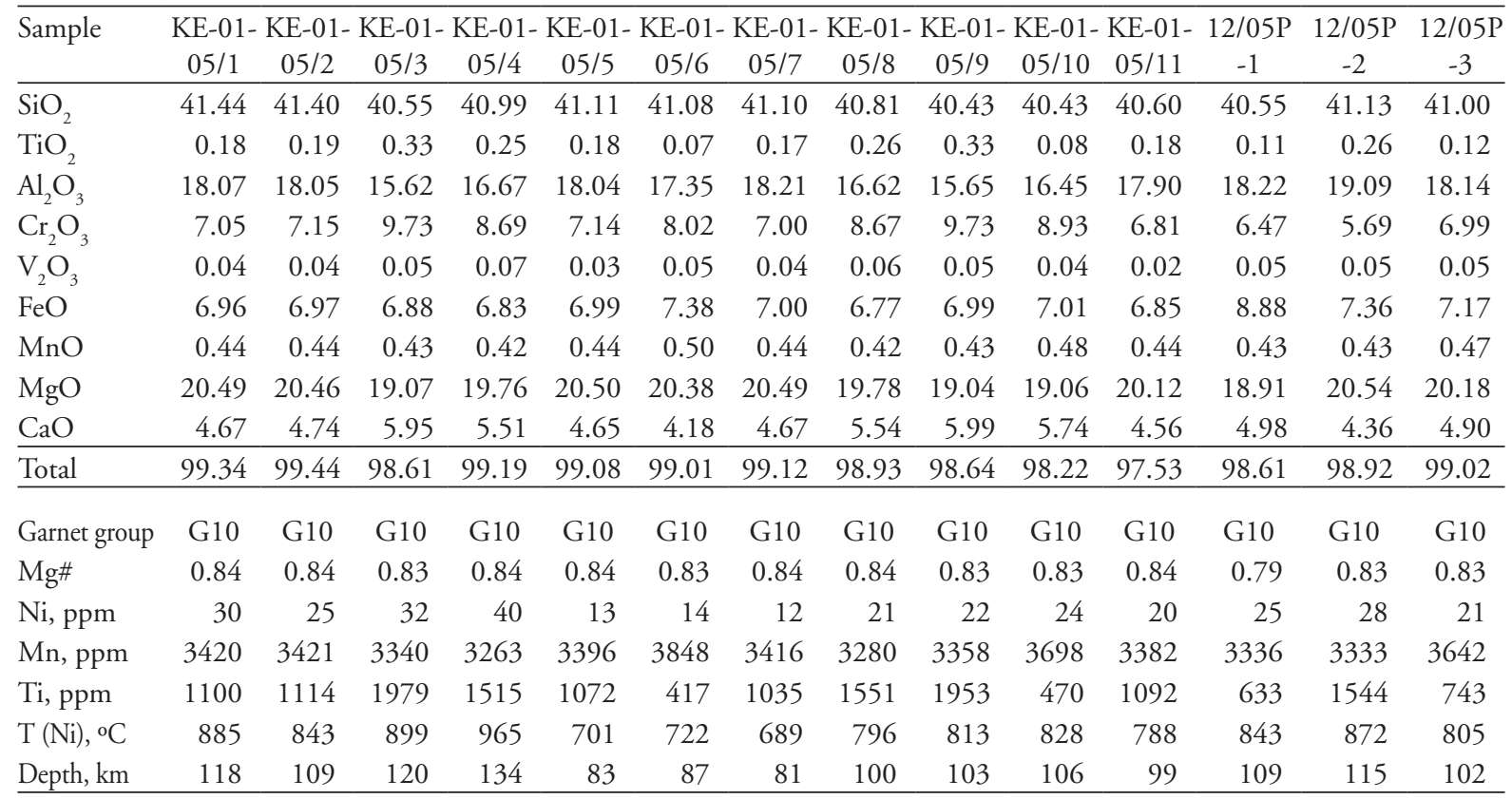

\begin{tabular}{|c|c|c|c|c|c|c|c|c|c|c|c|c|c|c|}
\hline Sample & $\begin{array}{c}12 / 05 \mathrm{P}- \\
4 \\
\end{array}$ & $\begin{array}{c}12 / 05 \mathrm{P}- \\
5 \\
\end{array}$ & $\begin{array}{c}12 / 05 \mathrm{P}- \\
6 \\
\end{array}$ & $\begin{array}{c}12 / 05 \mathrm{P}- \\
7 \\
\end{array}$ & $\begin{array}{c}12 / 05 \mathrm{P}- \\
8 \\
\end{array}$ & $\begin{array}{c}12 / 05 \mathrm{P}- \\
9 \\
\end{array}$ & $\begin{array}{c}12 / 05 \mathrm{P}- \\
10\end{array}$ & $\begin{array}{c}\text { KE-01- } \\
05 / 12 \\
\end{array}$ & $\begin{array}{c}\text { KE-01- } \\
05 / 13 \\
\end{array}$ & $\begin{array}{c}\text { KE-01- } \\
05 / 14 \\
\end{array}$ & $\begin{array}{c}\text { KE-01- } \\
05 / 15 \\
\end{array}$ & $\begin{array}{c}\text { KE-01- } \\
05 / 16 \\
\end{array}$ & $\begin{array}{c}\text { KE-01- } \\
05 / 17 \\
\end{array}$ & $\begin{array}{r}\text { KE- } 01- \\
05 / 18 \\
\end{array}$ \\
\hline $\mathrm{SiO}_{2}$ & 40.51 & 41.45 & 40.94 & 41.15 & 40.99 & 41.70 & 41.17 & 40.97 & 40.85 & 40.63 & 41.13 & 40.81 & 40.93 & 40.85 \\
\hline $\mathrm{TiO}_{2}$ & 0.03 & 0.09 & 0.12 & 0.12 & 0.11 & 0.02 & 0.12 & 0.26 & 0.41 & 0.41 & 0.15 & 0.41 & 0.16 & 0.41 \\
\hline $\mathrm{Al}_{2} \mathrm{O}_{3}$ & 15.79 & 18.38 & 18.00 & 18.06 & 18.07 & 19.73 & 18.15 & 17.98 & 16.41 & 16.35 & 17.44 & 16.60 & 17.42 & 16.48 \\
\hline $\mathrm{Cr}_{2} \mathrm{O}_{3}$ & 9.99 & 6.88 & 7.05 & 7.03 & 6.97 & 5.30 & 6.93 & 6.59 & 8.54 & 8.53 & 7.62 & 8.46 & 7.59 & 8.50 \\
\hline $\mathrm{V}_{2} \mathrm{O}_{3}$ & 0.04 & 0.05 & 0.02 & 0.03 & 0.02 & 0.04 & 0.03 & 0.06 & 0.05 & 0.05 & 0.05 & 0.06 & 0.05 & 0.05 \\
\hline $\mathrm{FeO}$ & 6.79 & 6.37 & 7.14 & 7.14 & 6.75 & 7.00 & 7.07 & 7.84 & 7.12 & 7.22 & 7.59 & 7.15 & 7.55 & 7.25 \\
\hline $\mathrm{MnO}$ & 0.43 & 0.40 & 0.47 & 0.47 & 0.40 & 0.41 & 46 & 0.47 & .45 & 0.44 & 0.47 & 0.44 & 0.47 & 0.44 \\
\hline $\mathrm{MgO}$ & 19.44 & 21.27 & 20.01 & 20.04 & 20.49 & 22.54 & 20.03 & 19.31 & 19.12 & 19.05 & 18.93 & 19.22 & 18.90 & 19.11 \\
\hline $\mathrm{CaO}$ & 5.78 & 4.22 & 4.90 & 4.90 & 4.80 & 2.19 & 4.90 & 5.29 & 6.12 & 6.08 & 5.91 & 6.15 & 5.93 & 6.10 \\
\hline Total & 98.80 & 99.11 & 98.65 & 98.94 & 98.60 & 98.93 & 98.86 & 98.77 & 99.07 & 98.76 & 99.29 & 99.30 & 99.00 & 99.19 \\
\hline $\begin{array}{l}\text { Garnet } \\
\text { group }\end{array}$ & G10 & G10 & G10 & G10 & G10 & G10 & G10 & G9 & G9 & G9 & G9 & G9 & G9 & G9 \\
\hline $\mathrm{Mg} \#$ & 0.84 & 0.86 & 0.83 & 0.83 & 0.84 & 0.85 & 0.83 & 0.81 & 0.83 & 0.82 & 0.82 & 0.83 & 0.82 & 0.82 \\
\hline $\mathrm{Ni}, \mathrm{ppm}$ & 1 & 14 & 25 & 10 & 28 & 20 & 39 & 9 & 12 & 35 & 32 & 26 & 15 & 21 \\
\hline Mn, ppm & 3363 & 3063 & 3650 & 3651 & 3066 & 3178 & 3596 & 3607 & 3461 & 3442 & 3600 & 3386 & 3627 & 3431 \\
\hline $\mathrm{Ti}, \mathrm{ppm}$ & 195 & 510 & 745 & 745 & 677 & 116 & 722 & 1535 & 2458 & 2449 & 925 & 2476 & 944 & 2429 \\
\hline $\mathrm{T}(\mathrm{Ni}),{ }^{\circ} \mathrm{C}$ & 371 & 722 & 836 & 665 & 865 & 788 & 954 & 638 & 689 & 924 & 899 & 851 & 733 & 796 \\
\hline Depth, km & 30 & 87 & 108 & 76 & 113 & 99 & 131 & 72 & 81 & 125 & 120 & 111 & 89 & 100 \\
\hline
\end{tabular}


Table 2. cont. Chemical composition of pyropes from Ermakovsky kimberlite, Terskii Coast field of the southern Kola region (wt.\%)

\begin{tabular}{|c|c|c|c|c|c|c|c|c|c|c|c|}
\hline Sample & $\begin{array}{c}\text { KE-01- } \\
05 / 19\end{array}$ & $\begin{array}{c}\mathrm{KE}-01- \\
05 / 20\end{array}$ & $\begin{array}{c}\text { KE-01- } \\
05 / 21\end{array}$ & $\begin{array}{c}\mathrm{KE}-01- \\
05 / 22 \\
\end{array}$ & $\begin{array}{c}\text { KE-01- } \\
05 / 23\end{array}$ & $\begin{array}{c}\text { KE-01- } \\
05 / 24\end{array}$ & $\begin{array}{c}12 / 05 \mathrm{P}- \\
11 \\
\end{array}$ & $\begin{array}{c}12 / 05 \mathrm{P}- \\
12 \\
\end{array}$ & $\begin{array}{c}12 / 05 \mathrm{P}- \\
13 \\
\end{array}$ & $\begin{array}{c}12 / 05 \mathrm{P}- \\
14\end{array}$ & $\begin{array}{c}12 / 05 \mathrm{P}- \\
15\end{array}$ \\
\hline $\mathrm{SiO}_{2}$ & 40.75 & 40.65 & 40.94 & 40.57 & 40.82 & 40.55 & 41.18 & 41.00 & 41.15 & 41.39 & 40.83 \\
\hline $\mathrm{TiO}_{2}$ & 0.41 & 0.40 & 0.30 & 0.41 & 0.16 & 0.15 & 0.31 & 0.14 & 0.27 & 0.15 & 0.10 \\
\hline $\mathrm{Al}_{2} \mathrm{O}_{3}$ & 16.57 & 16.46 & 17.44 & 16.45 & 17.51 & 17.30 & 19.27 & 18.11 & 19.51 & 19.58 & 16.88 \\
\hline $\mathrm{Cr}_{2} \mathrm{O}_{3}$ & 8.40 & 8.56 & 7.65 & 8.47 & 7.61 & 7.53 & 5.09 & 6.96 & 4.87 & 5.09 & 8.53 \\
\hline $\mathrm{V}_{2} \mathrm{O}_{3}$ & 0.05 & 0.06 & 0.07 & 0.06 & 0.05 & 0.06 & 0.04 & 0.06 & 0.05 & 0.05 & 0.05 \\
\hline $\mathrm{FeO}$ & 7.16 & 7.20 & 7.19 & 7.16 & 7.60 & 7.53 & 8.24 & 7.35 & 7.30 & 7.39 & 6.78 \\
\hline $\mathrm{MnO}$ & 0.44 & 0.45 & 0.43 & 0.44 & 0.47 & 0.47 & 0.45 & 0.46 & 0.43 & 0.42 & 0.45 \\
\hline $\mathrm{MgO}$ & 19.19 & 19.15 & 19.39 & 19.08 & 18.93 & 18.84 & 19.86 & 19.62 & 20.23 & 20.46 & 19.29 \\
\hline $\mathrm{CaO}$ & 6.12 & 6.15 & 5.76 & 6.09 & 5.95 & 5.86 & 4.91 & 5.51 & 4.98 & 4.85 & 5.99 \\
\hline Total & 99.09 & 99.08 & 99.17 & 98.73 & 99.10 & 98.29 & 99.35 & 99.21 & 98.79 & 99.38 & 98.90 \\
\hline Garnet group & G9 & G9 & G9 & G9 & G9 & G9 & G9 & G9 & G9 & G9 & G9 \\
\hline Mg\# & 0.83 & 0.83 & 0.83 & 0.83 & 0.82 & 0.82 & 0.81 & 0.83 & 0.83 & 0.83 & 0.84 \\
\hline $\mathrm{Ni}, \mathrm{ppm}$ & 16 & 43 & 22 & 29 & 7 & 38 & 14 & 9 & 30 & 24 & 13 \\
\hline $\mathrm{Mn}, \mathrm{ppm}$ & 3424 & 3455 & 3361 & 3428 & 3656 & 3623 & 3516 & 3598 & 3324 & 3268 & 3468 \\
\hline $\mathrm{Ti}$, ppm & 2432 & 2406 & 1812 & 2429 & 971 & 882 & 1863 & 841 & 1588 & 878 & 609 \\
\hline $\mathrm{T}(\mathrm{Ni}),{ }^{\circ} \mathrm{C}$ & 743 & 982 & 813 & 879 & 607 & 948 & 722 & 652 & 885 & 828 & 701 \\
\hline Depth, km & 90 & 137 & 103 & 116 & 67 & 130 & 87 & 74 & 118 & 106 & 83 \\
\hline
\end{tabular}

Table 3. Representative chemical analyses of the mantle-derived chrome diopsides from the Quaternary sediments of the Kola region (wt.\%)

\begin{tabular}{lcrrrrrrrrrrr}
\hline Sample & PL-2.02 & \multicolumn{1}{c}{$\mathrm{R}-4$} & \multicolumn{1}{c}{$\mathrm{S}-7$} & \multicolumn{1}{c}{$8 \_6$} & \multicolumn{1}{c}{ PL-3-4a } & $\mathrm{PL}-3-2$ & \multicolumn{1}{c}{$\mathrm{x}-3$} & \multicolumn{1}{c}{$\mathrm{x}-1$} & \multicolumn{1}{c}{$8 \_9$} & \multicolumn{1}{c}{$\mathrm{S}-5$} & $\mathrm{BL}-24 / 2$ & BL54 \\
\hline $\mathrm{SiO}_{2}$ & 53.69 & 53.50 & 52.86 & 54.56 & 53.24 & 53.68 & 52.49 & 52.23 & 53.13 & 53.37 & 54.83 & 55.05 \\
$\mathrm{TiO}_{2}$ & 0.24 & 0.09 & 0.37 & 0.00 & 0.39 & 0.17 & 0.36 & 0.37 & 0.00 & 0.03 & 0.22 & 0.27 \\
$\mathrm{Al}_{2} \mathrm{O}_{3}$ & 1.34 & 1.80 & 2.02 & 2.34 & 2.48 & 2.99 & 3.14 & 3.23 & 3.30 & 3.80 & 1.35 & 1.69 \\
$\mathrm{Cr}_{2} \mathrm{O}_{3}$ & 1.45 & 1.11 & 1.37 & 1.29 & 1.15 & 1.12 & 1.20 & 0.98 & 1.16 & 1.27 & 1.66 & 0.52 \\
$\mathrm{FeO}$ & 3.56 & 3.16 & 4.01 & 3.15 & 4.47 & 2.20 & 4.72 & 4.67 & 4.68 & 2.66 & 2.60 & 2.92 \\
$\mathrm{MnO}$ & 0.09 & 0.10 & 0.09 & 0.05 & 0.12 & 0.06 & 0.10 & 0.11 & 0.08 & 0.09 & 0.09 & 0.04 \\
$\mathrm{MgO}$ & 17.38 & 16.58 & 16.14 & 15.89 & 15.24 & 15.61 & 15.31 & 15.02 & 15.05 & 14.48 & 17.88 & 18.09 \\
$\mathrm{CaO}$ & 20.54 & 22.55 & 20.92 & 22.00 & 21.75 & 22.52 & 21.20 & 21.61 & 22.94 & 22.37 & 20.52 & 21.67 \\
$\mathrm{~K} \mathrm{O}$ & 0.00 & 0.00 & 0.00 & 0.00 & 0.00 & 0.00 & 0.00 & 0.00 & 0.00 & 0.00 & 0.01 & 0.00 \\
$\mathrm{Na}{ }_{2} \mathrm{O}$ & 0.96 & 0.78 & 1.04 & 0.80 & 0.75 & 1.14 & 0.89 & 0.90 & 0.61 & 1.46 & 0.87 & 0.44 \\
$\mathrm{NiO}$ & 0.00 & 0.00 & 0.08 & 0.00 & 0.05 & 0.06 & 0.00 & 0.00 & 0.00 & 0.06 & 0.12 & 0.03 \\
\hline $\mathrm{Total}$ & 99.25 & 99.67 & 98.90 & 100.08 & 99.64 & 99.55 & 99.41 & 99.12 & 100.95 & 99.59 & 100.15 & 100.72 \\
\hline $\mathrm{Ca} /(\mathrm{Ca}+\mathrm{Mg})$ & 0.46 & 0.49 & 0.48 & 0.50 & 0.51 & 0.51 & 0.50 & 0.51 & 0.52 & 0.53 & 0.45 & 0.46 \\
$\mathrm{Mg} \#$ & 0.90 & 0.90 & 0.88 & 0.90 & 0.86 & 0.93 & 0.85 & 0.85 & 0.85 & 0.91 & 0.92 & 0.92 \\
& $\mathrm{~g}$ & $\mathrm{~g}$ & $\mathrm{~g}$ & $\mathrm{~g}$ & $\mathrm{~g}$ & $\mathrm{~g}$ & $\mathrm{~g}$ & $\mathrm{~g}$ & $\mathrm{~g}$ & $\mathrm{~g}$ & $\mathrm{~g}$ & $\mathrm{~g}$ \\
$\mathrm{~T}\left({ }^{\circ} \mathrm{C}\right)$ & 1075 & 837 & 967 & 951 & 934 & 703 & 950 & 876 & 820 & 509 & 1133 & 1105 \\
$\mathrm{P}(\mathrm{kbar})$ & 44 & 30 & 35 & 30 & 28 & 20 & 26 & 23 & 20 & 15 & 44 & 37 \\
\hline
\end{tabular}

$\mathrm{d}$ - peridotitic chrome diopside from stability field of diamond; $\mathrm{g}$ - peridotitic chrome diopside from stability field of graphite; e - eclogitic clinopyroxene.

P-T parameters for peridotitic diopsides are calculated using Nimis \& Taylor (2000) thermobarometer. 
Table 3. cont. Representative chemical analyses of the mantle-derived chrome diopsides from the Quaternary sediments of the Kola region (wt.\%)

\begin{tabular}{lcccccccccccc}
\hline Sample & $\mathrm{R}-4$ & PL-27 & PP-2.02 & E1.1.02 & \multicolumn{1}{c}{$\mathrm{Sh}-5$} & BL-23/2/2 & PL-27-1 & PL-27-2 & PL-27-3 & PL-23-1 & PL-23-3 & PL-23-4 \\
\hline $\mathrm{SiO}_{2}$ & 53.81 & 55.08 & 53.92 & 54.12 & 53.42 & 54.67 & 54.02 & 55.08 & 54.00 & 54.40 & 54.56 & 54.27 \\
$\mathrm{TiO}_{2}$ & 0.11 & 0.04 & 0.27 & 0.11 & 0.12 & 0.27 & 0.15 & 0.04 & 0.14 & 0.09 & 0.08 & 0.06 \\
$\mathrm{Al}_{2} \mathrm{O}_{3}$ & 1.77 & 0.37 & 1.27 & 0.39 & 4.01 & 2.19 & 0.54 & 0.37 & 0.41 & 0.40 & 0.44 & 0.37 \\
$\mathrm{Cr}_{2} \mathrm{O}_{3}$ & 1.23 & 1.23 & 1.47 & 1.36 & 1.15 & 1.64 & 1.02 & 1.22 & 1.00 & 1.52 & 1.17 & 1.90 \\
$\mathrm{FeO}$ & 3.40 & 2.11 & 3.74 & 1.96 & 2.73 & 4.37 & 2.27 & 2.11 & 2.22 & 1.96 & 2.16 & 1.82 \\
$\mathrm{MnO}$ & 0.08 & 0.05 & 0.11 & 0.07 & 0.13 & 0.12 & 0.04 & 0.05 & 0.05 & 0.06 & 0.05 & 0.02 \\
$\mathrm{MgO}$ & 16.27 & 18.07 & 17.29 & 17.90 & 14.98 & 19.21 & 18.08 & 18.07 & 18.50 & 18.25 & 18.03 & 18.16 \\
$\mathrm{CaO}$ & 21.97 & 21.85 & 20.20 & 21.92 & 21.17 & 17.26 & 22.44 & 21.85 & 22.46 & 22.06 & 22.61 & 21.85 \\
$\mathrm{~K} \mathrm{O}$ & 0.02 & 0.02 & 0.02 & 0.01 & 0.02 & 0.00 & 0.01 & 0.02 & 0.00 & 0.00 & 0.01 & 0.00 \\
$\mathrm{Na} 2 \mathrm{O}$ & 0.65 & 0.59 & 0.84 & 0.65 & 1.46 & 0.89 & 0.62 & 0.59 & 0.56 & 0.76 & 0.68 & 0.86 \\
$\mathrm{NiO}$ & 0.17 & 0.03 & 0.04 & 0.01 & 0.05 & 0.05 & 0.00 & 0.03 & 0.02 & 0.03 & 0.08 & 0.07 \\
\hline $\mathrm{Total}$ & 99.48 & 99.44 & 99.17 & 98.50 & 99.24 & 100.67 & 99.19 & 99.43 & 99.36 & 99.53 & 99.87 & 99.38 \\
\hline $\mathrm{Ca} /(\mathrm{Ca}+\mathrm{Mg})$ & 0.49 & 0.47 & 0.46 & 0.47 & 0.50 & 0.39 & 0.47 & 0.47 & 0.47 & 0.46 & 0.47 & 0.46 \\
$\mathrm{Mg} \#$ & 0.89 & 0.94 & 0.89 & 0.94 & 0.91 & 0.89 & 0.93 & 0.94 & 0.94 & 0.94 & 0.94 & 0.95 \\
& $\mathrm{~g}$ & $\mathrm{~d}$ & $\mathrm{~g}$ & $\mathrm{~d}$ & $\mathrm{~g}$ & $\mathrm{~g}$ & $\mathrm{~d}$ & $\mathrm{~d}$ & $\mathrm{~d}$ & $\mathrm{~d}$ & $\mathrm{~d}$ & $\mathrm{~d}$ \\
$\mathrm{~T}\left({ }^{\circ} \mathrm{C}\right)$ & 964 & 1081 & 1124 & 1015 & 832 & 1300 & 952 & 1081 & 983 & 998 & 941 & 992 \\
$\mathrm{P}(\mathrm{kbar})$ & 34 & 57 & 45 & 54 & 21 & 43 & 51 & 57 & 55 & 53 & 53 & 52 \\
\hline
\end{tabular}

$\mathrm{d}$ - peridotitic chrome diopside from stability field of diamond; $\mathrm{g}$ - peridotitic chrome diopside from stability field of graphite; e - eclogitic clinopyroxene,

P-T parameters for peridotitic diopsides are calculated using Nimis \& Taylor (2000) thermobarometer.

\begin{tabular}{|c|c|c|c|c|c|c|c|c|c|c|c|c|c|c|}
\hline Sample & $\begin{array}{l}\text { PL- } \\
20\end{array}$ & $\begin{array}{c}\text { PL-23- } \\
1\end{array}$ & $\begin{array}{c}\text { PL-23- } \\
5\end{array}$ & $\begin{array}{c}\text { PL-23- } \\
6\end{array}$ & $\begin{array}{c}\text { PL-16- } \\
02\end{array}$ & $\begin{array}{c}\text { PL-27 } \\
-4\end{array}$ & $\begin{array}{c}\text { PL-22- } \\
02\end{array}$ & $\begin{array}{c}\text { PL-24- } \\
02\end{array}$ & SH-2 & P-256 & $\begin{array}{c}\text { PL-23- } \\
8\end{array}$ & $\begin{array}{c}\text { PL-19- } \\
02\end{array}$ & $\begin{array}{c}\text { PL-2- } \\
02-2\end{array}$ & $\begin{array}{c}\text { PL-3- } \\
5\end{array}$ \\
\hline $\mathrm{SiO}_{2}$ & 52.86 & 52.9 & 53.36 & 54.19 & 53.15 & 53.66 & 54.91 & 54.38 & 54.8 & 54.75 & 54.35 & 54.74 & 53.85 & 53.89 \\
\hline $\mathrm{TiO}_{2}$ & 0.28 & 0.28 & 0.23 & 0.12 & 0.28 & 0.14 & 0.04 & 0.10 & 0.09 & 0.35 & 0.04 & 0.13 & 0.06 & 0.13 \\
\hline $\mathrm{Al}_{2} \mathrm{O}_{3}$ & 2.66 & 2.60 & 1.86 & 1.82 & 2.24 & 1.99 & 0.36 & 0.44 & 0.40 & 1.90 & 4.47 & 4.46 & 4.39 & 5.25 \\
\hline $\mathrm{Cr}_{2} \mathrm{O}_{3}$ & 1.02 & 1.64 & 1.72 & 0.64 & 1.53 & 0.84 & 1.01 & 1.02 & 1.09 & 1.08 & 0.70 & 0.73 & 0.74 & 0.66 \\
\hline $\mathrm{FeO}$ & 4.55 & 3.37 & 3.09 & 3.44 & 3.57 & 3.19 & 1.98 & 2.13 & 2.07 & 3.30 & 3.76 & 3.52 & 3.79 & 4.31 \\
\hline $\mathrm{MnO}$ & 0.13 & 0.01 & 0.02 & 0.10 & 0.05 & 0.06 & 0.02 & 0.00 & 0.05 & 0.08 & 0.07 & 0.02 & 0.00 & 0.07 \\
\hline $\mathrm{MgO}$ & 15.31 & 16.51 & 16.8 & 16.94 & 16.84 & 17.39 & 18.47 & 18.42 & 17.98 & 16.97 & 13.93 & 14.00 & 14.03 & 13.59 \\
\hline $\mathrm{CaO}$ & 20.70 & 20.94 & 20.77 & 21.64 & 21.05 & 21.79 & 22.6 & 22.56 & 21.93 & 19.39 & 19.2 & 19.29 & 19.38 & 18.87 \\
\hline $\mathrm{K}_{2} \mathrm{O}$ & 0.01 & 0.01 & 0.00 & 0.00 & 0.00 & 0.02 & 0.02 & 0.00 & 0.00 & 0.04 & 0.00 & 0.01 & 0.01 & 0.00 \\
\hline $\mathrm{Na}_{2} \mathrm{O}$ & 1.49 & 1.19 & 1.26 & 1.10 & 0.94 & 0.86 & 0.59 & 0.61 & 0.55 & 1.86 & 3.05 & 2.92 & 3.05 & 2.89 \\
\hline $\mathrm{NiO}^{2}$ & 0.03 & 0.02 & 0.01 & 0.08 & 0.01 & 0.03 & 0.02 & 0.02 & 0.05 & 0.03 & 0.07 & 0.04 & 0.05 & 0.04 \\
\hline Total & 99.04 & 99.47 & 99.12 & 100.07 & 99.66 & 99.97 & 100.02 & 99.68 & 99.01 & 99.75 & 99.64 & 99.86 & 99.35 & 99.70 \\
\hline $\mathrm{Ca} /(\mathrm{Ca}+\mathrm{Mg})$ & 0.49 & 0.48 & 0.47 & 0.48 & 0.47 & 0.47 & 0.47 & 0.47 & 0.47 & 0.45 & 0.50 & 0.50 & 0.50 & 0.50 \\
\hline $\mathrm{Mg \#}$ & 0.86 & 0.90 & 0.91 & 0.90 & 0.89 & 0.91 & 0.94 & 0.94 & 0.94 & 0.90 & 0.87 & 0.88 & 0.87 & 0.85 \\
\hline & $\mathrm{g}$ & $\mathrm{g}$ & g & g & g & g & d & d & d & d & $\mathrm{e}$ & e & $\mathrm{e}$ & e \\
\hline $\mathrm{T}\left({ }^{\circ} \mathrm{C}\right)$ & 861 & 947 & 968 & 921 & 1002 & 954 & 996 & 970 & 1068 & 1068 & & & & \\
\hline $\mathrm{P}$ (kbar) & 30 & 31 & 37 & 37 & 33 & 33 & 59 & 55 & 56 & 65 & & & & \\
\hline
\end{tabular}

$\mathrm{d}$ - peridotitic chrome diopside from stability field of diamond; $\mathrm{g}$ - peridotitic chrome diopside from stability field of graphite; e - eclogitic chrome diopside.

P-T parameters for peridotitic diopsides are calculated using Nimis \& Taylor (2000) thermobarometer. 

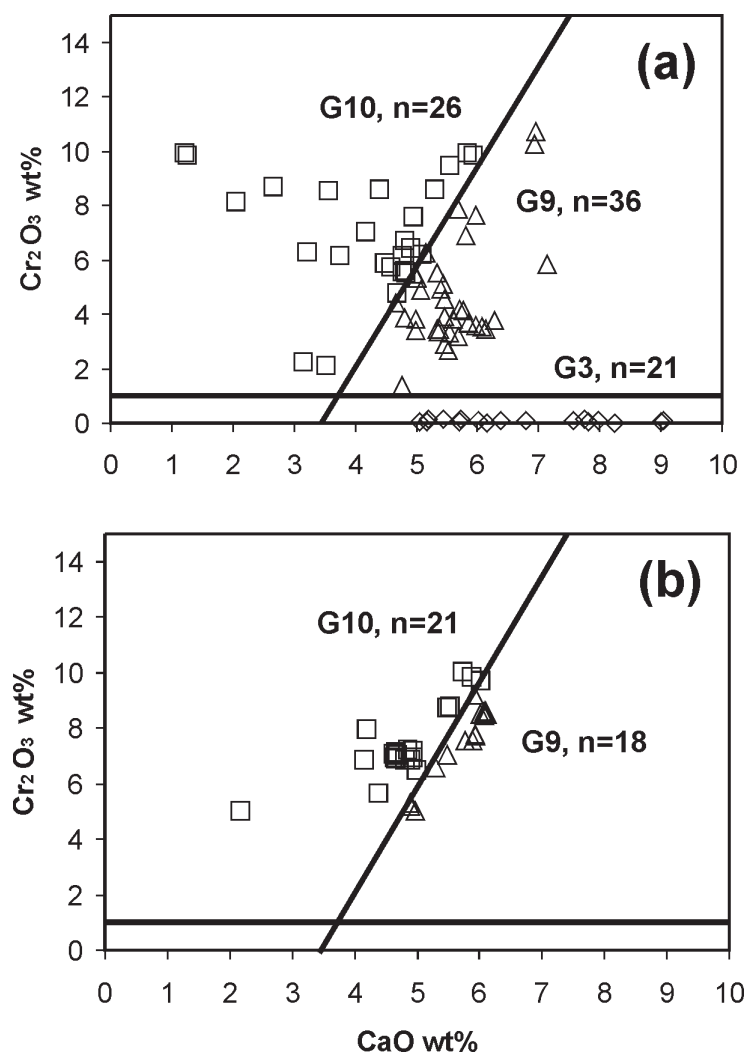

Fig. 2. $\mathrm{CaO}$ vs $\mathrm{Cr}_{2} \mathrm{O}_{3}$ diagram for pyropes from the south-eastern part of Kola region (a) and from Ermakovsky kimberlite (b). G9 (Iherzolitic pyropes), GI0 (harzburgitic pyropes) and G3 (eclogitic pyrope-almandines) fields are outlined based on the systems of Sobolev et al. (1973), Sobolev (1974) and Gurney (1984).

with lower contents of $\mathrm{MgO}(10-16 \%), \mathrm{Cr}_{2} \mathrm{O}_{3}$ ( $<1 \%)$, and elevated $\mathrm{FeO}$ (10-18\%), apparently originating from eclogites. Similar garnets are often found in deep seated xenoliths from alkaline and alkaline-ultrabasic dykes and pipes of the region, and rarely in the Ermakovsky kimberlite (Arzamastsev et al., 1988; Vetrin \& Kalinkin, 1992; Kalinkin et al., 1993). A portion of the G3 garnets with the highest $\mathrm{MgO}$ and $\mathrm{FeO}$ contents (c. $10 \%$ of total amount) could be related to the association of magnesium-ferrous eclogites. This type of diamondiferous xenoliths are found in kimberlites of the Arkhangelsk province (Bogatikov, 2000). Nevertheless to avoid ambiguity the entire G3 group is excluded from the following
P-T calculations, as crustal eclogites are known in the Kola region (Mitrofanov, 1996).

As a summary of chemical-genetic discrimination, the quantitative distribution of pyropes from Quaternary sediments of the south-eastern part of Kola region ( $80 \%$ of diamondiferous peridotitic associations, $10 \%$ of diamondiferous magnesium-ferrous eclogitic association, $10 \%$ of diamond-associated pyropes) seems to be very similar to kimberlites of Zolotitza field from Arkhangelsk province (75\%, $11 \%$ and $11 \%$, respectively, Bogatikov, 2000)). Thus it can be speculated that discoveries will be made in south-eastern Kola of the same high-grade kimberlitic pipes as in the Arkhangelsk province.

Garnets from Ermakovsky kimberlite show a different distribution of the chemical-genetic groups: G9 and G10 pyropes have equal proportions, G3 pyropes are not found (Fig. 2b), suggesting a distinctly different mantle source for these pyropes compared to those from the south-eastern Kola region.

Ni-thermometry (Ryan et al., 1996) on pyropes from south-eastern Kola gives a range of temperatures of $650-1250^{\circ} \mathrm{C}$, corresponding to a sampling interval of c. 75-190 km (Fig. 3). For depth estimates the regional geotherm of Kukkonen \& Peltonen (1999) was used. From the distribution of the different pyrope groups and their trace element compositions, a stratified structure to the southern Kola Craton lithospheric mantle is inferred: G10-pyropes are nearly absent in the shallow mantle horizon (layer A: 75-110 $\mathrm{km}$ ) which is the main source of G9-pyropes, whereas a deeper mantle horizon between 110 and $190 \mathrm{~km}$ (layer B) has contributed abundant G10-pyropes. The existence of abundant harzburgitic pyropes is taken as evidence that this mantle is Archean in nature. Based on $\mathrm{Mg} \#$ of the pyropes, the composition of this mantle lies midway between that from the more fertile Kuopio-Kaavi mantle at craton edge and the depleted craton core mantle seen at Kuhmo-Kuusamo (Lehtonen et al., 2004; O'Brien et al., 2003, 2006; Peltonen \& Brügmann, 2006; Zozulya et al., 2008). Overall, c. $16 \%$ of the pyropes are derived from the stability field of diamond, i.e. from a depth of $140-190 \mathrm{~km}$. 

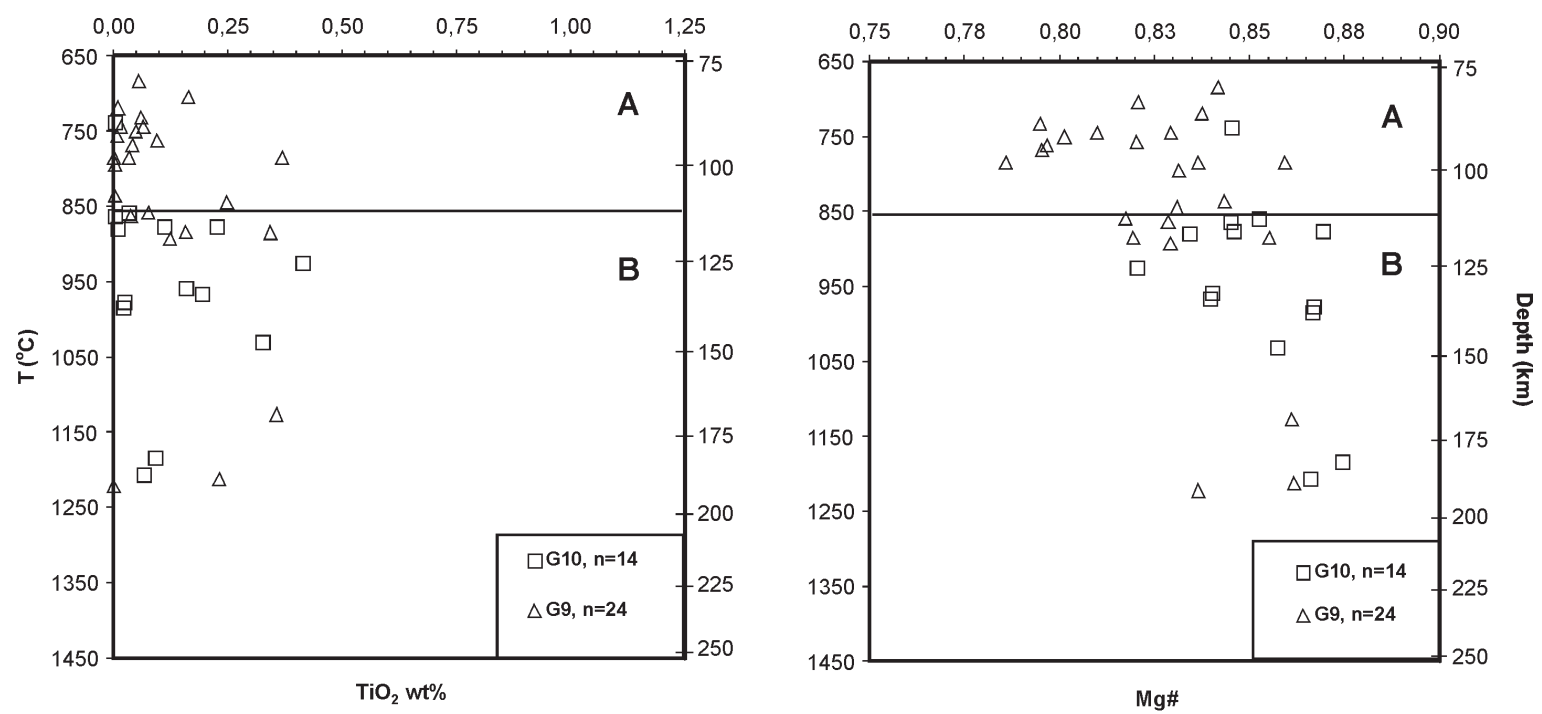

Fig. 3. Calculated temperature and depth versus $\mathrm{TiO}_{2}$ content and $\mathrm{Mg \#}$ for pyropes from south-eastern Kola region. $\mathrm{A}=$ lithospheric mantle layer with Iherzolitic pyropes, $\mathrm{B}=$ layer with Iherzolitic and harzburgitic pyropes.
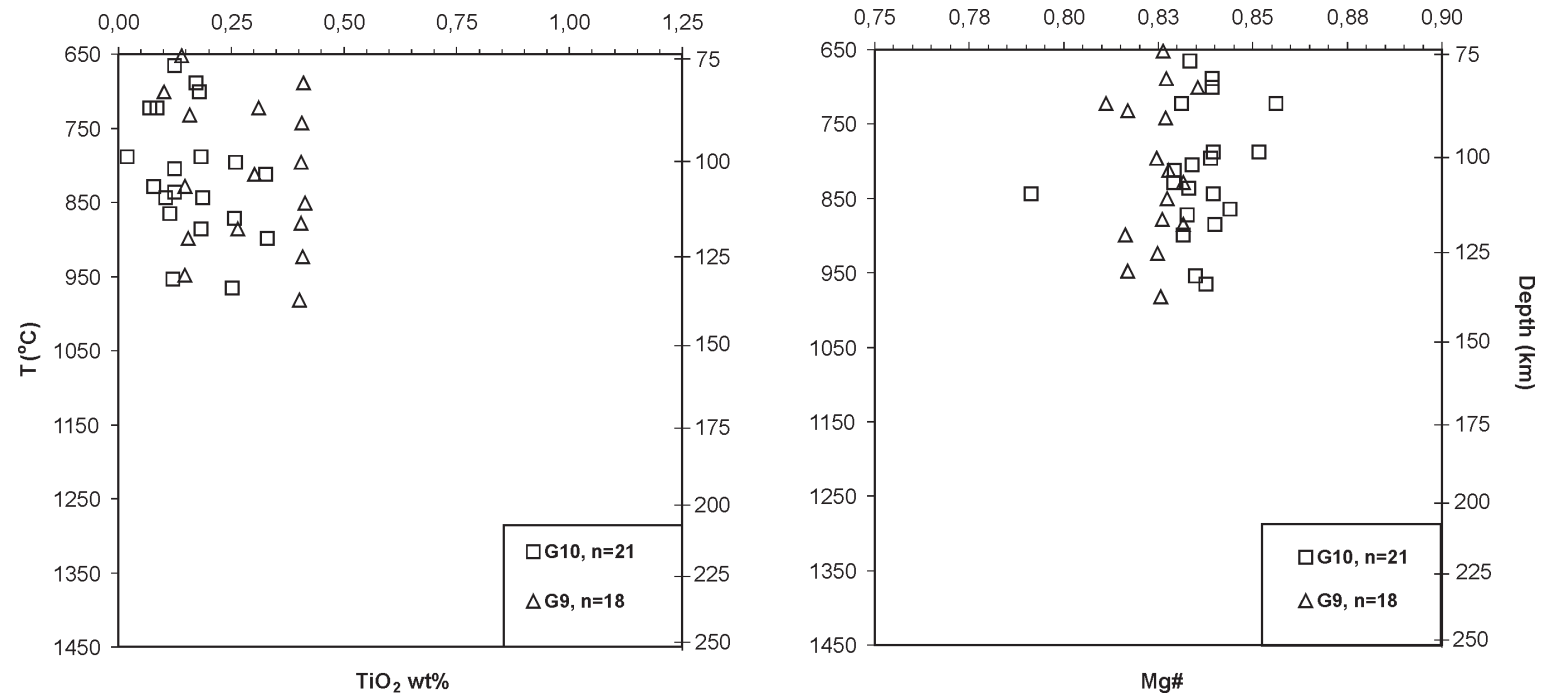

Fig. 4. Calculated temperature and depth versus $\mathrm{TiO}_{2}$ content and $\mathrm{Mg \#}$ for pyropes from Ermakovsky kimberlite in Terskii Coast field (southern Kola region).

Pyropes from the Ermakovsky kimberlite show a temperature range of $650-1000{ }^{\circ} \mathrm{C}$, corresponding to a sampling interval of c. $75-140 \mathrm{~km}$ (Fig. 4), in accordance with the low diamond grades determined for these pipes. The temperature values obtained for pyropes are comparable to determinations on chro- mites from Ermakovsky kimberlite $\left(820-1080{ }^{\circ} \mathrm{C}\right.$, c. 20 samples), calculated with the $\mathrm{Zn}$-thermometer of Ryan et al. (1996). These pyrope data show a different lithosphere mantle composition beneath the area; one that is relatively uniform in its harzburgite-lherzolite distribution. This could be explained by 
the spatial proximity of the Ermakovsky area to the Kandalaksha graben of the Belomorian rift system where Riphean (c. 1.2 Ga) and Paleozoic (c. 0.36 Ga) activations of the rift may have altered the originally stratified Archean lithospheric mantle. Additionally, melt metasomatism is confirmed for this mantle, based on higher $\mathrm{TiO}_{2}$ contents in pyropes (average value $0.21 \mathrm{wt} . \%$ ) relative to those from the southeastern area (average value $0.11 \%$ ).

\subsection{Mineral chemistry and mantle source rocks of the pyroxenes}

Diopsides from the Kola region show the following variations: $\mathrm{MgO}$ (10-18 wt.\%), $\mathrm{Cr}_{2} \mathrm{O}_{3}(0.3-3.6$ wt.\%), $\mathrm{Al}_{2} \mathrm{O}_{3}$ (0.3-6.3 wt.\%), $\mathrm{Na}_{2} \mathrm{O}$ (0.3-5.2 wt.\%), $\mathrm{TiO}_{2}(0.0-0.45$ wt.\%). These variations are rather wide, due to the presence of both eclogitic and peridotitic associations which form distinct compositional groups. The diopside group with higher $\mathrm{Na}_{2} \mathrm{O}$ and $\mathrm{Al}_{2} \mathrm{O}_{3}$, and lower $\mathrm{MgO}$ and $\mathrm{CaO}$, is related to the eclogite association. Diopsides of similar composition have been reported in eclogitic xenoliths from alkaline-ultrabasic rocks of the Kola region (Arzamastsev et al., 1988; Vetrin \& Kalinkin, 1992). The second group has elevated $\mathrm{MgO}, \mathrm{CaO}$ and $\mathrm{Cr}_{2} \mathrm{O}_{3}$, and lower $\mathrm{Na}_{2} \mathrm{O}$ and $\mathrm{Al}_{2} \mathrm{O}_{3}$, similar to diopsides of peridotitic associations from kimberlites and nondiamondiferous alkaline-ultrabasic pipes. Peridoditic diopsides were discriminated using the following parameters: $\mathrm{Al}_{2} \mathrm{O}_{3}<4.5$ wt. $\%, \mathrm{Cr}_{2} \mathrm{O}_{3}>0.5$ wt. $\%, \mathrm{Na}_{2} \mathrm{O}<2$ wt. $\%$ and $\mathrm{MgO}>15$ wt.\% (Zozulya et al., 2008). The sampled sediments with peridotitic diopsides often contained other kimberlitic indicator minerals, especially pyrope, chrome spinel and/or olivine (Gavrilenko et al., 2000; Zozulya et al., 2008).

$\mathrm{P}-\mathrm{T}$ determinations on peridotitic chrome diopsides using the single-grain thermobarometer of $\mathrm{Ni}$ mis and Taylor (2000) imply that the majority of grains from south-eastern Kola fall into the graphite stability field within 20-45 kbar and 700-1300 ${ }^{\circ} \mathrm{C}$ (Fig. 5a). These grains apparently originated from non-diamondiferous ultramafic xenoliths in alkaline-ultramafic dykes of the region, widely manifest- ed to the west of the sampling area and transported by Scandinavian glaciers (Fig. 1). Nevertheless, c. $15 \%$ of south-eastern Kola diopsides yield values of 40-60 kbar and 700-1100 ${ }^{\circ} \mathrm{C}$ and indicate derivation from the diamond-stability field. Diopsides from south-western Kola yield P-T estimates in the range of 45-65 kbar and $850-1100^{\circ} \mathrm{C}$ (Fig. 5a), and dominantly plot in the diamond-stability field. Diopsides from the central Kola yield P-T values of 20-55 kbar and $700-1150{ }^{\circ} \mathrm{C}$ (Fig. 5b), with c. $20 \%$ derived from the diamond-stability field. Diopsides from the northern Kola region, in contrast, yield P-T values of $20-45 \mathrm{kbar}$ and $600-1300{ }^{\circ} \mathrm{C}$ (Fig. 5c), all falling within the graphite-stability field. The maximum depth of xenocryst sampling varies from up to 200 $\mathrm{km}$ in the southern Kola, to $170 \mathrm{~km}$ in central Kola, to only $140 \mathrm{~km}$ in the northern Kola region.

Diopsides from different depth horizons vary in composition. Pyroxenes from the diamond-stability field are characterized by lower contents of $\mathrm{TiO}_{2}$ (typical values $0.01-0.15$ wt. $\%), \mathrm{Al}_{2} \mathrm{O}_{3}(0.3-0.7$ wt. \%), $\mathrm{FeO}$ (1.8-2.3 wt.\%), $\mathrm{Na}_{2} \mathrm{O}$ (0.6-0.8 wt.\%), higher

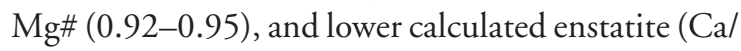
$(\mathrm{Ca}+\mathrm{Mg})=0.45-0.47)$. Pyroxenes of similar composition have been reported from Yakutia and Arkhangelsk kimberlites associated with diamonds (Sobolev, 1974; Bogatikov, 2000). Pyroxenes from the graphite-stability field have higher $\mathrm{TiO}_{2}(0.14-0.38$ wt.\%), $\mathrm{Al}_{2} \mathrm{O}_{3}$ (1.7-2.7 wt.\%), FeO (3.0-4.5 wt.\%), $\mathrm{Na}_{2} \mathrm{O}(0.9-1.5$ wt.\%), lower $\mathrm{Mg \#} \mathrm{(0.86-0.91),} \mathrm{and}$ relatively higher calculated enstatite $(\mathrm{Ca} /(\mathrm{Ca}+\mathrm{Mg})$ $=0.47-0.50)$. Diopsides similar to these have been found in peridotitic xenoliths from alkaline-ultrabasic rocks of the Kola region (Arzamastsev et al., 1988; Vetrin \& Kalinkin, 1992).

The P-T values for chrome diopsides thus imply significant regional variations in heat flow. Within the southern part of the Kola, adjacent to the Kandalaksha graben, the chrome diopside data (clusters 2 and 3 on Fig. 5a) plot between the 36 and $46 \mathrm{~mW} / \mathrm{m}^{2}$ model geotherms of Pollack and Chapman (1977). Importantly, towards the east and towards the west, away from the Kandalaksha graben, the lithosphere appears to become thicker and the heat flow corre- 

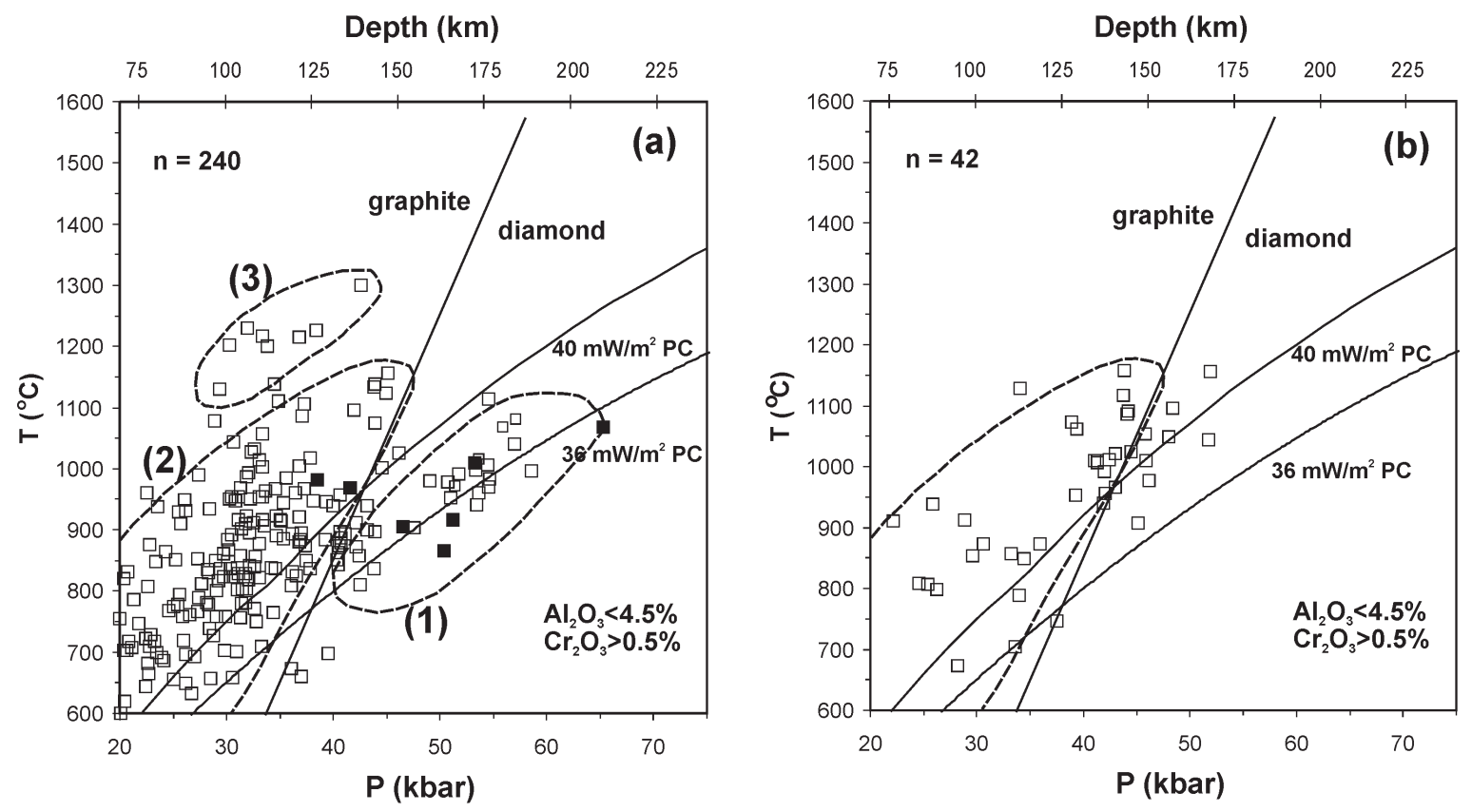

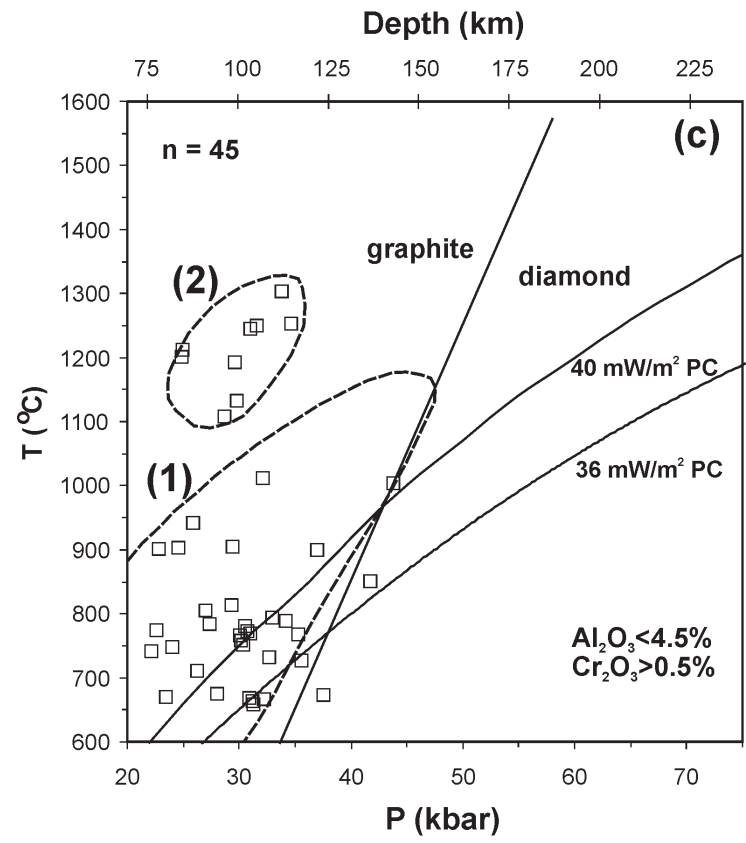

sponds to a cool cratonic model geotherm of 35-38 $\mathrm{mW} / \mathrm{m}^{2}$ (cluster 1 on Fig. 5a). The central Kola, in turn, is characterized by a more elevated heat flow of c. $38-44 \mathrm{~mW} / \mathrm{m}^{2}$. The highest heat flow values (up to $50 \mathrm{~mW} / \mathrm{m}^{2}$, cluster 2 on Fig. $5 \mathrm{c}$ ), are observed in the northernmost Kola Craton, adjacent to the Barents rift system.
Fig. 5. Calculated P-T parameters for peridotitic chrome diopsides from Quaternary sediments of the Kola region. (a) south-eastern (open squares) and south-western (solid squares) areas. (b) central area. (c) northern area. PC - model geotherm of Pollack \& Chapman (1977). Clusters I, 2, 3 outline the different pyroxene geotherms.

\section{Discussion and conclusions}

The Archean Kola craton is prospective for diamonds based on structural tectonic conditions, discoveries of diamond-bearing kimberlites (the Ermakovsky pipes in the southern Kola region) and the presence of kimberlitic indicator minerals and diamonds in Quater- 


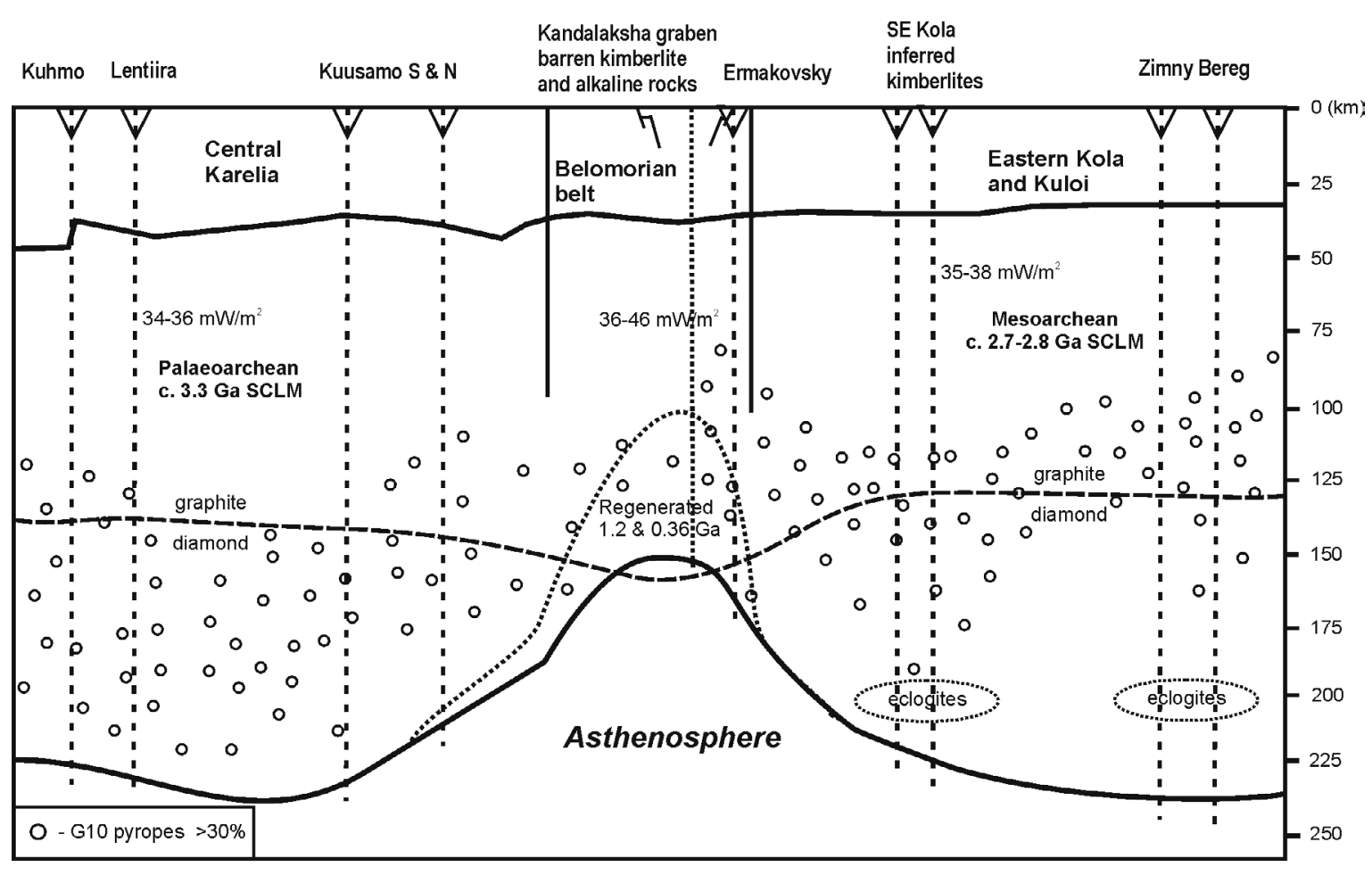

Fig. 6. Simplified geological cross-section of the subcontinental lithospheric mantle (SCLM) along the NE Karelian craton - Kandalaksha graben - SE Kola craton transect with kimberlitic occurrences shown. Arkhangelsk data after Lehtonen et al. (2009).

nary sediments. The results of this work demonstrate that undiscovered kimberlites probably exist in the southern and central parts of Kola.

Compositions of garnet xenocrysts from the Ermakovsky kimberlites are in agreement with the low-grade of the pipes. The samples contain G9 and G10 garnets in equal proportions but their $\mathrm{Ni}$ -temperatures reveal a relatively shallow sampling interval of the mantle (c. $75-140 \mathrm{~km}$ ), dominantly within the graphite-stability field. The shallow extent of the lithosphere and the evidence of melt metasomatism in the pyrope data could be explained by the spatial proximity of the Ermakovsky area to the Belomorian rift system.

Our data indicate that the more "cratonic" southeastern and south-western parts of the Kola region have higher potential for diamondiferous kimberlitic magmatism than the northern and central part of the Kola craton (Fig.1). Next to the Kandalaksha graben in the south, where the Ermakovsky kimberlites are located, the P-T data from single-Cpx thermobarometry ranges widely between the 36 and $46 \mathrm{~mW} / \mathrm{m}^{2}$ conductive model geotherms. Towards the east and the west the lithosphere increases in thickness up to $200 \mathrm{~km}$ and the heat flow corresponds to a cool cratonic geotherm of $35-38 \mathrm{~mW} / \mathrm{m}^{2}$, favorable for diamond deposits. The central Kola has again a higher heat flow of c. $38-44 \mathrm{~mW} / \mathrm{m}^{2}$ and a lithosphere thickness of around $170 \mathrm{~km}$. The heat flow reaches its maximum value (c. $\left.50 \mathrm{~mW} / \mathrm{m}^{2}\right)$ in the northern Kola, next to the Barents rift system, where the lithosphere is also at its thinnest (c. $140 \mathrm{~km}$ ).

Combining the dataset provided here with previously published xenolith and xenocryst thermobarometry from the Karelian and Kola cratons (Lehtonen et al., 2004; O’Brien et al., 2003, 2006; Peltonen \& Brügmann, 2006; Zozulya et al., 2008) allows the following structure of the subcontinental lithospheric mantle (SCLM) to be outlined along a NE Karelian craton - Kandalaksha graben - SE Kola 
craton transect (Fig. 6). For the major part of the Karelian craton and for the SE part of the Kola craton, deep lithospheric roots are inferred. The roots are of Archean age and the cratonic geotherm is cool. Asthenosphere uplift is observed in the north-easternmost part of Karelia craton (Kuusamo area) and in southern Kola craton (Ermakovsky area, adjacent to Kandalaksha graben) up to 180 and $140-150 \mathrm{~km}$, correspondingly. The lithospheric mantle of this segment is characterized by elevated heat flow values. Apparently, regeneration of asthenospheric mantle occurred here during 1.2 and $0.36 \mathrm{Ga}$ rifting. This area is characterized by abundant occurrences of rift-related alkaline and ultrabasic-alkaline dykes and pipes and has low to no diamond potential.

The diamond prospectivity of the south-eastern and south-western Kola is further supported by the following observations:

1) The south-eastern samples contain high numbers of diamond-indicative G10 garnets, comparable to the highly diamondiferous kimberlites of the Zolotitsa field in the Arkhangelsk province. In addition, the samples contain abundant eclogitic G3 pyropes that are absent in the diamond-poor Ermakovsky kimberlites, suggesting distinctly different mantle sources for these two localities.

2) Ni-thermometry of the pyropes in the southeastern region indicates a wide range of sampling (70-190 km) of the lithospheric mantle, with c. $16 \%$ of the pyropes derived from the diamond stability field between $140-190 \mathrm{~km}$.

3) Despite the fact that the Cr-diopside population of the south-eastern samples is dominated by apparently non-kimberlitic grains, a significant proportion of the diopsides (15\%) still yields PT parameters within the diamond stability field. This holds true also for the major portion of the Cr-diopsides from south-western Kola. Significantly, the pyroxenes plotting inside the diamond stability field have similar compositions to pyroxenes associated with diamonds from Yakutia and Arkhangelsk kimberlites.

\section{Acknowledgments}

The study was partly supported by the Department of Earth Science, Russian Academy of Sciences (Programs $6 \& 8$ ). We thank A. Arzamastsev for providing chromite samples, and O. Korsakova and V. Kalatchev for assistance in field-work. Bo Johanson and Lassi Pakkanen are thanked for help with the microprobe. We also wish to thank E. Sharkov for his helpful comments and the comprehensive review by $\mathrm{H}$. Downes, which improved the manuscript considerably.

\section{References}

Arzamastsev, A.A., Kaverina, V.A. \& Polezhaeva, L.I., 1988. The dyke rocks of Khibiny massif and its surroundings. Kola Sci. Centre, Apatity, 86p. (in Russian).

Arzamastsev, A.A. \& Dahlgren, S. 1994. Plutonic mineral assemblages in Paleozoic dikes and explosion pipes of the Alkaline Province of the Baltic Shield. Geochemistry International 31 (3), 57-68.

Baluev, A.S., 2006. Geodynamics of the Riphean Stage in the Evolution of the Northern Passive Margin of the East European Craton. Geotectonics 40, 183-196.

Beard, A.D., Downes, H., Vetrin, V., Kempton, P.D. \& Maluski, H., 1996. Petrogenesis of Devonian lamprophyre and carbonatite minor intrusions, Kandalaksha Gulf (Kola Peninsula, Russia). Lithos 39, 93-119.

Beard, A.D., Downes, H., Hegner, E., Sablukov, S.M., Vetrin, V.R. \& Balogh, K., 1998. Mineralogy and geochemistry of Devonian ultramafic minor intrusions of the southern Kola Peninsula, Russia: implications for the petrogenesis of kimberlites and melilitites. Contributions to Mineralogy and Petrology 130, 288-303.

Beard, A.D., Downes, H., Mason, P.R.D. \& Vetrin, V.R., 2007. Depletion and enrichment processes in the lithospheric mantle beneath the Kola Peninsula (Russia): Evidence from spinel lherzolite and wehrlite xenoliths. Lithos 94, 1-24.

Bogatikov, O.A., (ed.) 2000. Arkhangelsk diamond province. Moscow State University, Moscow, 524p. (in Russian).

Downes, H., Balaganskaya, E., Beard, A., Liferovich, R., \& Demaiffe, D., 2005. Petrogenetic processes in the ultramafic, alkaline and carbonatitic magmatism in the Kola alkaline province: a review. Lithos 85, 48-75.

Gavrilenko, B.V., Mitrofanov, F.P., Zozulya, D.R., Chikiryov, I.V., Sorokhtin, N.O., Kalachyov, V.Yu. \& Basalaeva, V.I., 2000. Diamond placer prospectivity of the Kola region. Proceedings of Murmansk State Technical University 3 (2), 235-244. (in Russian).

Gurney, J.J., 1984. A correlation between garnets and diamonds. University of WA, Publ. 8, 143-166. 
Delenitzin, A.A., Gavrilenko, B.V., Serov, P.N. \& Bayanova, T.B., 2001. Sm-Nd and Rb-Sr age determination of Yermakovskaya-7 kimberlite pipe, Tersky bereg, Kola region. SVEKALAPKO and EUROPROBE project. 6th Workshop. Lammi, Finland, 29.11-2.12.2001. Abstract. University Oulu, Dep. Geophysics, Report No 24, p. 17 .

Hattestrand, C., Kolka, V. \& Stroeven A.P., 2007. The Keiva ice marginal zone on the Kola Peninsula, northwest Russia: a key component for reconstructing the palaeoglaciology of the northeastern Fennoscandian Ice Sheet. Boreas 36, 352-370.

Hirvas, H. 1977. Glacial transport in Finnish Lapland. In: Prospecting in areas of glaciated terrain 1977: papers presented at a symposium organized by the Institution of Mining and Metallurgy, with the cooperation of the Geological Survey of Finland and held in Helsinki, Finland, on 15-17 August, 1977. Institution of Mining and Metallurgy, 128-137.

Kalinkin, M.M., Arzamastsev, A.A. \& Polyakov, I.V., 1993. Kimberlites and related rocks of the Kola region. Petrology 1, 173-180.

Kukkonen, I.T. \& Peltonen P., 1999. Xenolith controlled geotherm for the central Fennoscandian Shield: implications for lithosphere-asthenosphere relations. Tectonophysics 304, 301-315.

Lehtonen, M.L., O’Brien, H.E., Peltonen, P., Johanson, B.S. \& Pakkanen, L.K., 2004. Layered mantle at the Karelian Craton margin: P-T of mantle xenocrysts and xenoliths from the Kaavi-Kuopio kimberlites, Finland. Lithos 77, 593-608.

Lehtonen, M.L., O’Brien, H.E., Peltonen, P., Kukkonen, I.T., Ustinov, V. \& Verzhak, V., 2009. Mantle xenocrysts from the Arkhangelskaya kimberlite (Lomonosov mine, NW Russia): Constraints on the composition and thermal state of the diamondiferous lithospheric mantle. Lithos 112S, 924-933.

Mitrofanov, F.P., (ed.) 1996. Geological map of the Kola region (North-Eastern Baltic Shield), 1:500000 Scale. Apatity.

Nimis, P. \& Taylor, W.R., 2000. Single clinopyroxene thermobarometry for garnet peridotites. Part I. Calibration and testing of a Cr-in-cpx barometer and an enstatitein-cpx thermometer. Contributions to Mineralogy and Petrology 139, 541-554.
O’Brien, H.E., Lehtonen, M.L., Spencer, R.G. \& Birnie, A.C., 2003. Lithospheric Mantle in Eastern Finland: 250 $\mathrm{km} 3 \mathrm{D}$ transect. In: Extended abstracts. 8th International Kimberlite Conference, Victoria, BC, Canada, June 22-27th 2003. 5 p.

O'Brien, H., Peltonen, P., Lehtonen, M. \& Zozulya, D., 2006. Mantle stratigraphy of the Karelian craton: a 620 $\mathrm{km}$ long 3-D cross section revealed by mantle-derived xenocryst chemistry. In: Kukkonen, I.T., Eklund, O., Korja, A., Korja, T., Pesonen, L.J. \& Poutiainen, M., (eds.) Lithosphere 2006, Fourth symposium on the structure, composition and evolution of the lithosphere in Finland, 9-10 Novermber 2006, in Espoo. Programme and extended abstracts. Institute of seismology, University of Helsinki, Report S-46, 137-139.

Peltonen, P. \& Brügmann, G., 2006. Origin of layered continental mantle (Karelian craton, Finland): geochemical and Re-Os isotope constraints. Lithos 89, 405-423.

Pollack, H.N. \& Chapman, D.S., 1977. On the regional variations of heat flow, geotherms and lithosphere thickness. Tectonophysics 38, 279-296.

Robinson, B.W. \& Graham, J., 1992. Advances in Electron Microprobe Trace Element Analysis. Journal of Computer-Assisted Microscopy 4, 263-265.

Ryan, C.G., Griffin, W.L. \& Pearson, N.J., 1996. Garnet geotherms: pressure-temperature data from $\mathrm{Cr}$-pyrope garnet xenocrysts in volcanic rocks. Journal of Geophysical Research 101, 5611-5625.

Sobolev, N.V., Lavrentiev, Yu.G., Pokhilenko, N.P. \& Usova, N.P., 1973. Chrome-rich garnets from the kimberlites of Yakutia and their paragenesis. Contributions to Mineralogy and Petrology 40, 39-52.

Sobolev, N.V., 1974. The deep seated inclusions in kimberlites and the problem of the upper mantle composition. Publishing House "Nauka", Novosibirsk, 264 p. (in Russian).

Vetrin, V.R. \& Kalinkin, M.M., 1992. Reconstruction of the processes of intracrustal and crustal-mantle magmatism and metasomatism (from the results of the investigation of deep seated xenoliths). Kola Sci. Centre, Apatity, 108 p. (in Russian).

Zozulya, D.R., Peltonen, P. \& O’Brien, H., 2008. Pyrope and $\mathrm{Cr}$-diopside as indicators of mantle structure and diamond depth facies in the Kola Region. Geology of Ore Deposits 50, 524-534. 